\title{
High Shear in Situ Exfoliation of 2D Gallium Oxide Sheets from Centrifugally Derived Thin Films of Liquid Gallium
}

Kasturi Vimalanathan, Timotheos Palmer, Zoe Gardner, Irene Ling, Soraya Rahpeima, Sait Elmas, Qiang Sun, Jason R. Gascooke, Christopher T Gibson, Jin Zou, Mats R. Anderson, Nadim Darwish, Colin Raston Submitted date: 11/05/2021 - Posted date: 12/05/2021

Licence: CC BY-NC-ND 4.0

Citation information: Vimalanathan, Kasturi; Palmer, Timotheos; Gardner, Zoe; Ling, Irene; Rahpeima, Soraya; Elmas, Sait; et al. (2021): High Shear in Situ Exfoliation of 2D Gallium Oxide Sheets from Centrifugally Derived Thin Films of Liquid Gallium. ChemRxiv. Preprint. https://doi.org/10.26434/chemrxiv.14571573.v1

Herein, we have explored the use of a microfluidics platform for the exfoliation and oxidation of liquid gallium into ultrathin sheets of gallium oxide under continuous flow condition. The novel method developed here takes advantage of the high mass transfer in liquids and has the potential for creating high yielding thin sheets of oxidised gallium with insulating properties as well as acts as an active catalyst in hydrogen evolution reactions. This highlights the potential utility of the sheets as an alternative to the expensive and scarce noble metal based electrocatalysts

File list (2)

High stress in situ exfoliation of 2D gallium oxide sheets fro... (7.20 MiB) view on ChemRxiv • download file 
High shear in situ exfoliation of 2D gallium oxide sheets from centrifugally derived thin films of liquid gallium

Kasturi Vimalanathan ${ }^{1 *}$, Timotheos Palmer ${ }^{1}$, Zoe Gardner ${ }^{1}$, Irene Ling ${ }^{2}$, Soraya Rahpeima ${ }^{1,3}$, Sait Elmas ${ }^{1}$, Jason R. Gascooke ${ }^{1}$,Christopher T. Gibson ${ }^{1,4}$, Qiang Sun ${ }^{5,6}$, Jin Zou ${ }^{5,6}$, Mats R. Anderson ${ }^{1}$, Nadim Darwish ${ }^{3}$ and Colin L. Raston ${ }^{1 *}$

${ }^{1}$ Flinders Institute for Nanoscale Science and Technology, College of Science and Engineering, Flinders University, Adelaide SA 5001, Australia

${ }^{2}$ School of Science, Monash University Malaysia, Jalan Lagoon Selatan, Bandar Sunway, 47500 Selangor, Malaysia

${ }^{3}$ School of Molecular and Life Sciences, Curtin Institute for Functional Molecule and Interfaces, Curtin University, Bentley, Western Australia 6102, Australia

${ }^{4}$ Flinders Microscopy and Microanalysis, College of Science and Engineering, Flinders University, Bedford Park, SA 5042, Australia

${ }^{5}$ Centre for Microscopy and Microanalysis, The University of Queensland, Brisbane, QLD 4072, Australia

${ }^{6}$ Materials Engineering, The University of Queensland, Brisbane, QLD 4072, Australia.

*Corresponding authors email: kasturi.vimalanathan@flinders.edu.au and colin.raston@flinders.edu.au

The potential of two dimensional (2D)-nanomaterials for tackling some of the fundamental issues facing humanity have emerged from the discovery of graphene. There is now a diversity of such materials with recent attention turning to $2 \mathrm{D}$ materials of the post-transition metal elements [1-3], in particular material derived from liquid metals and eutectic melts below $330{ }^{\circ} \mathrm{C}$ where processing is more flexible and in the temperature regime suitable for industry. These $2 \mathrm{D}$ materials can have useful properties, along with their corresponding oxidised material, which can be accessed directly from liquid metals. Centrifugal thinning of liquid metals with solvents under shear stress has potential in both facilitating exfoliation and oxidation of $2 \mathrm{D}$ materials, as well as controlling the formation of nanomaterials in general, with control over their morphology, phase and dimensions. This has been explored for liquid gallium using an angled vortex fluidic device (VFD) to fabricate ultrathin gallium oxide $\left(\mathrm{Ga}_{2} \mathrm{O}_{3}\right)$ sheets under continuous flow conditions. The new method takes advantage of high mass transfer in liquids, and has potential for creating $2 \mathrm{D}$ materials, and other nano-materials from liquid metals. Conducting atomic force microscopy for $\mathrm{Ga}_{2} \mathrm{O}_{3}$ nanosheets sandwiched between a platinum $\mathrm{AFM}$ tip and a $\mathrm{Si}$ substrate show that they form a highly insulating material reducing the current between the tip and the substrate by over three orders of magnitude. In addition, the $\mathrm{Ga}_{2} \mathrm{O}_{3}$ nanosheets have electrocatalytic activity for hydrogen evolution, with a Tafel slope of $39 \mathrm{mVdec}^{-1}$ revealing promoting effects of the surface oxidation (passivation layer). This highlights the potential utility of the ultrathin $\mathrm{Ga}_{2} \mathrm{O}_{3}$ nanosheets as an alternative to noble metal-based electrocatalysts. 
Over the past decade, 2D-nanomaterials have captured the attention of the scientific community, with their extraordinary properties and diversity in applications, seemingly having no boundaries. Nevertheless, there are shortcomings on gaining access to such material where scalability is addressed. This even applies to ubiquitous graphene, for the fabrication of high quality atomically thin sheets, in overcoming the strong van der Waals forces between the layers, and the limited dispersion prowess of the material in organic solvents. While graphene itself is a zero-band gap semiconductor and exhibits quasi metallic features, it exhibits limitations in applications primarily for use in devices and electronics [4, 5]. Having access to a library of 2D-nanomaterials with different properties offers scope for being able to tune the properties of the materials, and this has emerged as a driver for developing 2D-nanomaterials beyond graphene. A number of 2D materials that have recently emerged including transition metal dichalcogenides (TMDs), layered metal oxides, 2D inorganic compounds, hexagonal boron nitride ( $h$-BN), layered double hydroxides (LDHs), black phosphorus (BP) and other elemental main group metals and metalloids [5-15]. 2D-nanomaterials derived from liquid metals and/or eutectic melts of the metals, in particular, are envisaged to break new barriers, offering unique properties and potential in applications [16-23]. In addition, it is a significant advantage that any development as such needs to incorporate the principles of green chemistry to minimise the likelihood of any downstream issues, including adverse effects on the natural environment, with the process being readily scalable. We have recently explored the potential of mechanical energy induced within thin films of liquid in the variable angle vortex fluidic device (VFD), Figure 1a, for the synthesis and manipulation of nanomaterials, with the effectiveness of this type of processing in exfoliation of 2D materials, including graphene, $h$-BN and black phosphorus [24-26]. This approach has been successful for generating such material high in green chemistry credentials, without the need for surfactants and harsh chemicals, thereby minimising the waste stream, with the processing simple and low costing, using low molecular weight solvents and under continuous flow conditions. The angled VFD with an optimal tilt angle of $45^{\circ}$ (maximum cross vector between centrifugal and gravity) spun at high rotational speeds has remarkably diverse range of applications beyond the exfoliation of 2D materials, including scrolling of graphene directly from graphite flakes, laser assisted controlled slicing of carbon nanotubes, controlling chemical reactivity and selectivity, biocatalysis and probing the structure of self-organised systems [27-33], also incorporating green chemistry principles within the processing.

Herein, we report a simple biphasic method involving liquid gallium and an organic solvent for the in-situ generation and exfoliation of $\mathrm{Ga}_{2} \mathrm{O}_{3}$ sheets, yielding extremely thin sheets with lateral dimensions that are of the lateral dimensions of the bulk gallium layer centrifugally thinned. Gallium metal is a liquid at room temperature (m.p. $29.7{ }^{\circ} \mathrm{C}$ ) having negligible vapour pressure, with the solid state exhibiting both covalent and metallic bonding, and excellent electrical and thermal conductivity [34]. Also important is its low toxicity making the melt an attractive alternative to toxic mercury, and making it useful for a number of applications [35-39]. Similar to other liquid metals and alloys, gallium features a self-limiting thin oxide layer under ambient conditions, which partly contributes to the excellent properties the liquid metal has to offer at the metal air interface [40]. 2D Ga $\mathrm{O}_{3}$ sheets and nanoparticles have been previously reported using a variety of synthetic routes with the most common method involving high intensive cavitational energy, the squeezeprinting process whereby liquid gallium is forced between two substrates, and a nebulization technique with 
all resulting in surface oxidation, affording a nanometer thin layer of the oxide [20, 23, 40-42]. We hypothesised that in the VFD, this layer has the potential to be exfoliated in contact with a dynamic thin film of solvent, thereby exposing fresh metal for rapid oxidation under high mass transfer of oxygen into the film of metal, as a continuous flow exfoliation process, one layer of the $2 \mathrm{D}$ material at a time. Exfoliation of 2D material in the VFD is under controlled mechanical energy input in time and place and we also hypothesised that this would lead to uniform sized sheets of any exfoliated material. While metal oxide layers can be transferred to other surfaces [22, 40], strategies for direct transfer into a solvent for fabricating designer material are wanting. Also noteworthy is that the mechanical energy in a liquid over the liquid metal in the VFD can overcome the otherwise formation of a rigid mechanical shell (oxide layer) around the droplet of liquid metal, thereby attenuating its exfoliation.
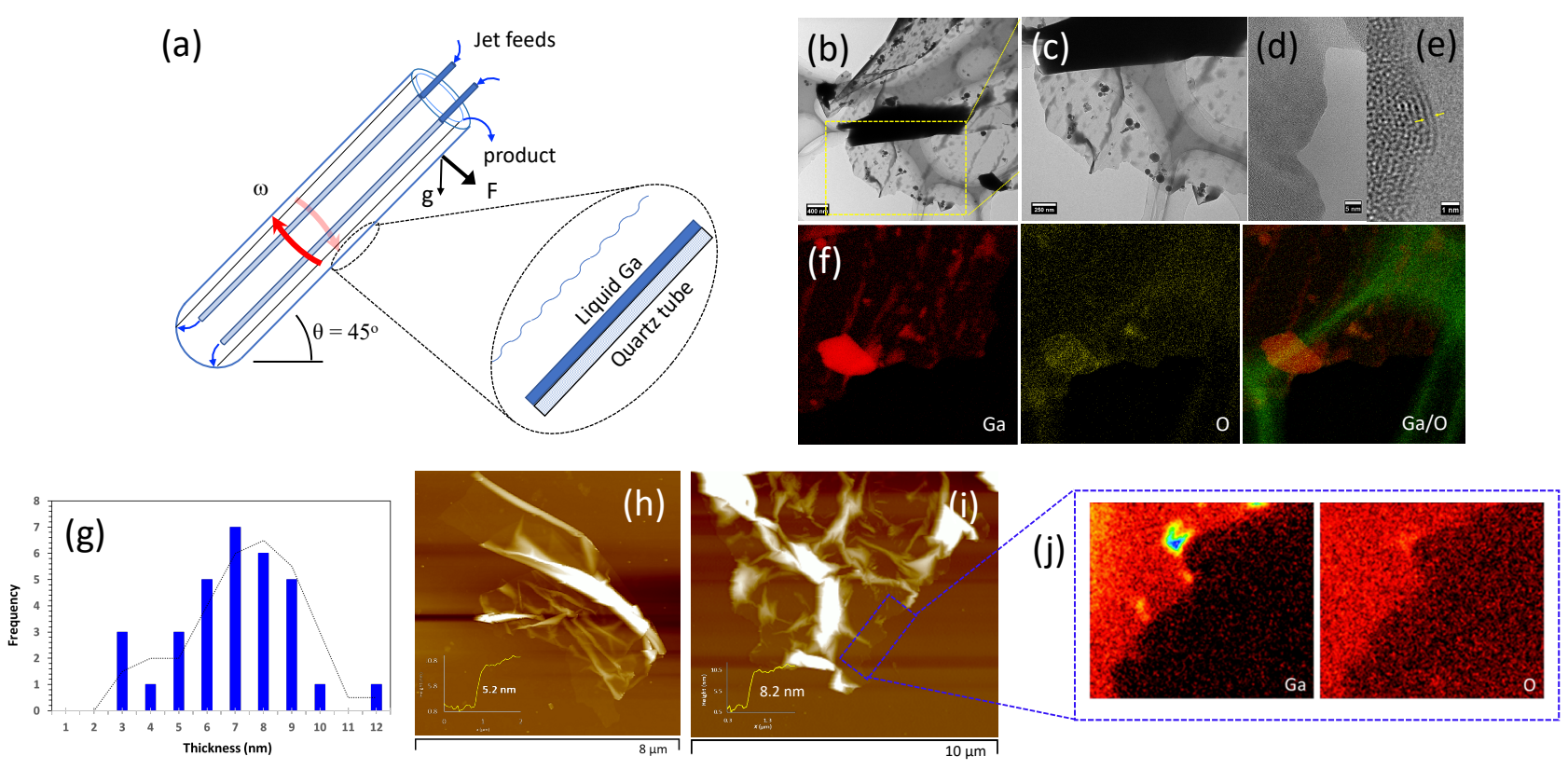

Fig.1 | VFD mediated fabrication of ultrathin $\mathrm{Ga}_{2} \mathrm{O}_{3}$ sheets. a Schematic of the VFD illustrating its salient features; b-c Bright field TEM images of an ultrathin sheet; d-e HRTEM images illustrating the lattice spacing of the sheets of $\sim 0.25 \mathrm{~nm}$ and $\mathbf{f}$ EDS maps of the corresponding sheet in $\mathbf{b} ; \mathbf{g}$ an average thickness distribution plot based on AFM measurements; $\mathbf{h}-\mathbf{i}$ AFM height image of the ultrathin sheets and $\mathbf{j}$ EDX map of the ultrathin sheet confirming the presence of $\mathrm{Ga}$ and $\mathrm{O}$ on the surface of the area corresponding to the section in $\mathrm{i}$.

The VFD was the thin film platform of choice given its utility in the top down manipulation of a diverse range of nanomaterials, having exquisite control of morphology, size and dimensions of particles size to suit specific applications, for either exfoliation or exfoliation with scrolling of the 2D material as discussed above. The key difference now is that the processing is essentially a biphasic system with the source of the $2 \mathrm{D}$ material being surface oxidised liquid metal. Biphasic processing in the VFD thus far has been around immiscible solvents including water, for fabricating nanomaterials [43, 44] and the separation of proteins [45]. To put it into perspective, the VFD features a borosilicate or quartz glass tube rotating at high speed (usually rotated between 2000 and $9000 \mathrm{rpm}$ ) inclined relative to the horizontal position, which is typically optimal for a specific process at $45^{\circ}$. Liquid is continuously delivered to the bottom of the glass tube, which then forms a dynamic thin film, 
Fig. 1a. The VFD encompasses a number of unique operating features that allows for efficient mass transfer of gases, intense micromixing of the reaction mediums and rapid dissipation of heat within the system which in combination is highly effective for the exfoliation of the $\mathrm{Ga}_{2} \mathrm{O}_{3}$ nanosheets.

\section{Results and discussion}

Synthesis and characterization of the $\mathrm{Ga}_{2} \mathrm{O}_{3}$ sheets. The design of our processing method was established based on systematically exploring the process parameters of the VFD (rotational speed, $\omega$, and inclination angle, q), the choice of solvent, temperature of the system and residence time of liquid entering the tube and exiting at the top. Gallium solid was crushed in a mortar and pestle to finer powder particles prior to immersing in $1 \mathrm{~mL}$ of $n$-propanol (10 mg/mL). $n$-Propanol was the choice of solvent after careful optimisation with other polar and non-polar solvents within the green chemistry scope, also including dimethylformamide (DMF), toluene, $i$-propanol, methanol, $o$-xylene and mesitylene. The mixture was then heated on a hot plate to $c a 30$ ${ }^{\circ} \mathrm{C}$ for $\sim 60$ seconds to achieve a molten liquid which was then subsequently transferred to a VFD quartz tube (20 mm OD, $17.5 \mathrm{~mm}$ ID, $18.5 \mathrm{~cm}$ long). The VFD process was optimised by systematically varying the rotational speed (4000 rpm to $9000 \mathrm{rpm})$ and the inclination angle $\left(0^{\circ}\right.$ to $\left.90^{\circ}\right)$, with the optimised conditions $7000 \mathrm{rpm}$ and $\theta 45^{\circ}$ respectively. The optimised speed is consistent with mixing times experiments undertaken to gain insight into the nature of the fluid dynamics within the thin film [46]. We observed a minimum time taken for $n$-propanol to mix and form a uniform film at the $7000 \mathrm{rpm}$ speed (Supplementary Fig. S1) which corresponds to the presence of benefitial Coriolis and Faraday wave effects in the thin film [46]. We also carried out a control experiment using an in situ heating jacket, a 'plug and play' attachment on the VFD (Supplementary Fig. S2) and heated the system at $30^{\circ} \mathrm{C}$ and $50^{\circ} \mathrm{C}$ at the abovementioned optimised rotational speed and tilt angle. The heating jacket was used to avoid prior heating of gallium metal on a hotplate. However, no sheets were observed using this approach. To address scalability of the process, we translated these above conditions into a continuous flow processing platform, with the liquid gallium $(200 \mathrm{mg})$ placed at the base of the quartz VFD tube with then injection of $n$-propanol at an optimised flow rate of $0.5 \mathrm{~mL} / \mathrm{min}$. The gallium oxide sheets were produced in high yield $(\sim 60 \%)$ following recycling through of the solvent, $n$ propanol. Here we established proof of concept of the potential for scaling up using parallel arrays of VFDs, coupled with recycling of the solvent and the gallium metal, or the use of the larger diameter, $50 \mathrm{~mm}$ VFD.

Thus heating the gallium metal to a molten liquid on a hot plate at $30^{\circ} \mathrm{C}$ is important prior to processing in the VFD. The temperature of the hot plate was pivotal, with temperatures $>30^{\circ} \mathrm{C}$ showing no evidence of sheets

being formed, and with temperatures below the melting point of gallium also ineffective. After 30 minutes of processing, the sheets were collected, centrifuged $(\mathrm{g}=3.22)$ to remove any contamination and large particles/solids of unreacted gallium within the sample, and then dried for further characterization. Scanning electron microscopy (SEM) and atomic force microscopy (AFM) were initially used to establish the size, morphology and thickness of the sheets. Transmission electron microscopy (TEM), high resolution transmission electron microscopy (HRTEM), Raman scectroscopy, scanning auger, energy dispersive X-ray (EDX) mapping, X-ray photoelectron spectroscopy (XPS), and X-ray powder diffraction (XRD) analysis were then used to further understand the nature of the 2D-sheets. 
Gaining insight into the mechanism of formation and surface properties of these ultrathin sheets, involved the use of AFM tapping mode and TEM and HRTEM on samples deposited directly on $\mathrm{SiO}_{2} / \mathrm{Si}$ wafers and on holey carbon grids respectively. The ultrathin sheets were observed to be thin with wrinkles on the surface. TEM/EDS (energy dispersive x-ray mapping) and SEM/EDX mapping were used to determine the elemental composition of the sheets, establishing the presence of Ga and O (Figure 1f) whilst HRTEM of the sheets showed poor crystallinity with a $d$-spacing of $0.25 \mathrm{~nm}$ and amorphous characteristics (Figure b-e) [47]. The oxidised gallium at the surface of the ultrathin sheets (Figure 1) arises from processing under ambient conditions where there is high mass transfer of oxygen into the thin film of $n$-propanol in contact with the thin layer of gallium centrifugally pinned against the wall of the VFD tube. As previously noted, most liquid metals feature such a self-limiting oxide layer under ambient conditions. The high shear stress generated in the VFD at $7000 \mathrm{rpm}$ and $45^{\circ}$ tilt angle is effective in 'peeling off' individual layers of ultrathin $\mathrm{Ga}_{2} \mathrm{O}_{3}$ sheets in contact with liquid gallium through dispersion forces, with subsequent exposed gallium metal then forming another layer of oxidized metal, which is also exfoliated in the same way, and so on. The peeling of the sheets from the surface of the gallium is facilitated by upward flow in the spinning top high mass transport topological fluid flow $\mu \mathrm{m}$ in diameter or less [46]. This flow was established in the VFD from moulding of bismuth metal (m.p. $271.4^{\circ} \mathrm{C}$ ) which melts on the surface of the tube at room temperature by the mechanical energy induced by down flow of the spinning top flow [46].

AFM height images established the average thickness of the exfoliated sheets at $\sim 5-6 \mathrm{~nm}$ (calculated from an average distribution of 50 sheets) (Figure 1g). Given that a single oxide layer of $\mathrm{Ga}_{2} \mathrm{O}_{3}$ has a thickness $\sim 1 \mathrm{~nm}$ [47], the exfoliated $\mathrm{Ga}_{2} \mathrm{O}_{3}$ sheets generated in the VFD consist of approximately 5 to 6 layers of the oxide material. The rotational speed of $7000 \mathrm{rpm}$ and $45^{\circ}$ inclination angle was critical for high yield exfoliation, with all other combinations studied affording either no sheets or much lower yield, and thus the residence and shear stress are critically important. The low crystallinity and partial amorphous characteristics, as observed from the HRTEM images, Figure 1d and 1e, is related to the high rotational speed contributing towards the shorter residence time for the gallium to be within the reaction system and therefore limiting time for reorganisation of the crystal structure. This is consistent with literature whereby unalloyed gallium sheets tend to show amorphous characteristics specifically when fabricated over short time scales [40]. Raman mapping of an ultrathin sheet, (Supplementary Fig. S3), was carried out for bulk gallium (black line), as well as for $\mathrm{Ga}_{2} \mathrm{O}_{3}$ sheets (blue line). The bulk gallium gave a characteristic band at approximately $300 \mathrm{~cm}^{-1}$ corresponding to the symmetrical stretching of pairs of gallium atoms [48, 49]. The Raman spectra of the generated ultrathin sheets in the present study have several weak and broad bands attributed to the bending and stretching of the Ga-O bond [49]. In addition, there is a strong broad signal between $50 \mathrm{~cm}^{-1}$ and $400 \mathrm{~cm}^{-1}$ with a maximum peak at about $\sim 50-100 \mathrm{~cm}^{-1}$ (Supplementary Fig. S3). The typical characteristic band around $300 \mathrm{~cm}^{-1}$ for gallium metal was not observed, possibly due to the low structural order of the $\mathrm{Ga}_{2} \mathrm{O}_{3}$ lattice [49]. A slight background signal was also observed in the Raman spectra of $\mathrm{Ga}_{2} \mathrm{O}_{3}$ which could be due to luminescence effects [49] 
(a)
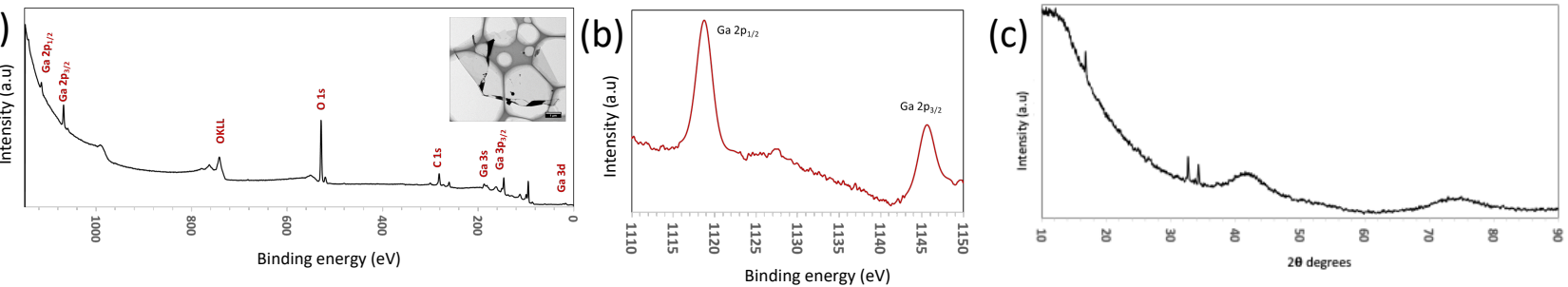

(d)
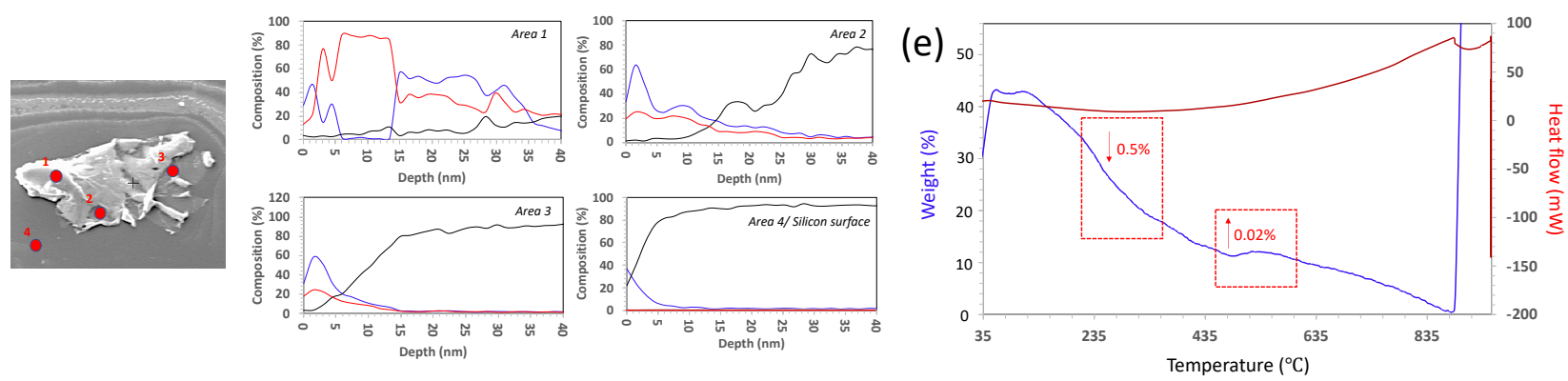

Fig. 2 | Surface characterization of the ultrathin $\mathrm{Ga}_{2} \mathrm{O}_{3}$ sheets. a XPS survey spectra of the $\mathrm{Ga}_{2} \mathrm{O}_{3}$ sheets; b High resolution XPS spectra showing two intense peaks at binding energies of $1145.03 \mathrm{eV}$ and 1118.13 $\mathrm{eV}$, which correspond to $2 \mathrm{p}_{3 / 2}$ and $2 \mathrm{p}_{1 / 2}$ states, respectively of the $\mathrm{Ga}_{2} \mathrm{O}_{3}$ sheets; $\mathbf{c}$ XRD pattern of the $\mathrm{Ga}_{2} \mathrm{O}_{3}$ sheets; $\mathbf{d}$ Auger depth profile analysis of an ultrathin $\mathrm{Ga}_{2} \mathrm{O}_{3}$ sheet (SEM image) and the corresponding depth profile graphs for 4 selected points (Area 1-4) (Figure legend: __ $\mathrm{Ga} ; \ldots \mathrm{O} ; \ldots \mathrm{Si}$ ) and e Thermal analysis using TGA/DSC at $10{ }^{\circ} \mathrm{C}$ of temperature increase under $\mathrm{N}_{2}$.

XPS measurements were carried out to further understand the surface composition and chemical states of the synthesized $\mathrm{Ga}_{2} \mathrm{O}_{3}$ sheets. The survey spectra showed the presence $\mathrm{Ga}, \mathrm{O}$ and traces amount of carbon in the sample. The high-resolution spectra of the Ga $2 p$ region afforded a doublet, Ga $2 p_{3 / 2}$ and Ga $2 p_{1 / 2}$ with the positions of the $\mathrm{Ga}_{2} \mathrm{O}_{3}$ sheets (Fig. 2a-b) peaks compared to the bulk gallium metal (Supplementary Fig. S3). The Ga $2 p_{3 / 2}$ and $\mathrm{Ga} 2 \mathrm{p}_{1 / 2}$ peaks are positioned at $1145.03 \mathrm{eV}$ and $1118.13 \mathrm{eV}$ respectively for bulk gallium with a shift by $0.75 \mathrm{eV}$ to a higher binding energy associated with the $\mathrm{Ga}-\mathrm{O}$ bonding in $\mathrm{Ga}_{2} \mathrm{O}_{3}$ [21] .

A further understanding of the surface of the sheets was gained through the use of surface sensitive Auger spectroscopy and corresponding depth profiling, to a depth of $40 \mathrm{~nm}$ (Fig. 2d). Three separate points on a single sheet were analysed with an additional control point on the silicon surface for comparison. The three separate points were chosen to represent distinct features observed on a sheet; Areas 1-2 were observed to be visibly thicker as a result of wrinkling and folding of the ultrathin sheets, with an approximate thickness of $\sim 40 \mathrm{~nm}$ and Area 3 as the thinnest area corresponding to a thickness of 6-7 $\mathrm{nm}$ and therefore there are several layers of exfoliated oxide layers in this region, as previously discussed [47]. Depth profiling of Areas 1-2 revealed gallium and oxygen on the top most layer up to a depth of $5 \mathrm{~nm}$. Interestingly, between 5-15 nm, the sheet was observed to contain predominantly gallium with no oxygen present followed by an increase in oxygen at depths between $15-35 \mathrm{~nm}$. This would suggest that there is a region which contains unoxidized gallium. Depth profiling beyond this showed a decrease in both gallium and oxygen. Similarly, for Area 3 as the thinnest region, gallium and oxygen are present on the uppermost layer up to a depth of $\sim 5 \mathrm{~nm}$ before a decrease in both gallium and oxygen content beyond $10 \mathrm{~nm}$ depth. These findings are important as they 
contribute towards the understanding of the mechanism of formation of these ultrathin $\mathrm{Ga}_{2} \mathrm{O}_{3}$ sheets, and in particular, suggests that although the thinnest layers present are completely oxidised, there also are areas of 'sandwiched' unoxidised gallium, specifically around regions with thickness greater than $7 \mathrm{~nm}$.

X-ray diffraction (XRD) was used to characterize the crystal phase, crystallinity and purity of the sheets. $\mathrm{Ga}_{2} \mathrm{O}_{3}$ can crystallize in a number of polymorphs including $\alpha-, \beta-, \gamma-, \delta$ - and $\varepsilon$ phases with the $\alpha$ - phase typically formed as the outer oxide layer in the liquid metal [50]. Amongst these polymorphs, the $\alpha$ - and $\beta$-phase are the most structurally documented with the $\beta$ - phase being the most thermodynamically stable [50]. All diffraction patterns for the $\mathrm{Ga}_{2} \mathrm{O}_{3}$ sheets could be indexed to $\alpha$-gallium oxide (Fig.2c) and no additional diffraction peaks of possible impurities here were detected. We attempted to relate the crystal structures of the $\alpha$ - and $\beta-\mathrm{Ga}_{2} \mathrm{O}_{3}$ (ICSD numbers: 27431 and 83645 respectively) to match the possible polymorph formed from the synthesized $\mathrm{Ga}_{2} \mathrm{O}_{3}$ sheets. $\alpha-\mathrm{Ga}_{2} \mathrm{O}_{3}$ crystallizes in the high symmetry $R \overline{3} c$ double hexagonal close-packed system (Supplementary Fig. S5) while $\beta-\mathrm{Ga}_{2} \mathrm{O}_{3}$ crystallizes in the $C 2 / m$ distorted double hexagonal closepacked system (Fig. 3b). Fingerprint plots generated from the Hirshfeld surface analysis can provide rapid visual means of discriminating the similarities and/or differences in atomic interactions between gallium and oxygen atoms, as well as summarizing the overall distribution of all close contacts quantitatively for both types of polymorph, Fig. $3 \mathrm{~b}$ and $3 \mathrm{e}$. This includes the variation in the percentage of Ga... O/O...Ga and O...O contacts in the Hirshfeld surfaces. The intermolecular contacts in both $\alpha$ - and $\beta-\mathrm{Ga}_{2} \mathrm{O}_{3}$ are overwhelmingly $\mathrm{Ga}$...O/O...Ga (either as donor or acceptor) with $79.6 \%$ and $74.2 \%$ respectively; while the remaining contribution to the Hirshfeld surface from O...O contacts $20.3 \%$ and $24.2 \%$ respectively, Fig. $3 \mathrm{c}$ and $3 \mathrm{f}$. Both polymorphs showed exceptionally small ranges of $\mathrm{Ga}$...O/O...Ga contacts, evident from the two narrow bands at the lower regions of $d_{\mathrm{e}}$ and $d_{\mathrm{i}}$ on the fingerprint plot. However, the $\alpha-\mathrm{Ga}_{2} \mathrm{O}_{3}$ showed more intense red and green streaks indicating that the majority of the contacts fall within the short range and can be rationalised in terms of more efficient packing amongst the atoms. O...O contacts are less dominant in the $\alpha-\mathrm{Ga}_{2} \mathrm{O}_{3}$, as judged by the sparseness and colour of the dots in the fingerprint plot. 


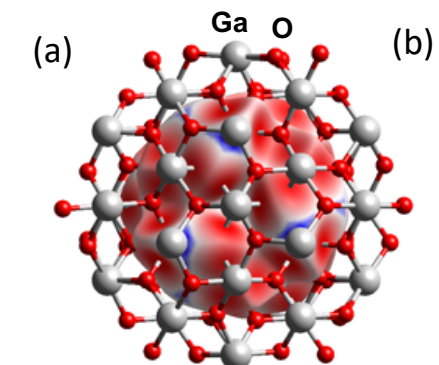

$R \overline{3} c$

(c)

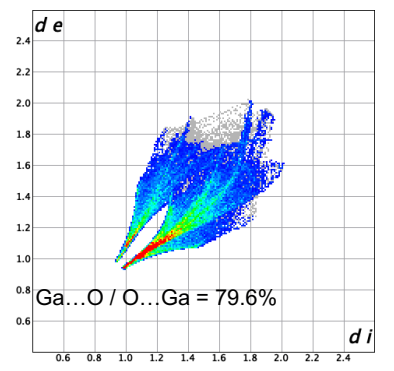

(b)
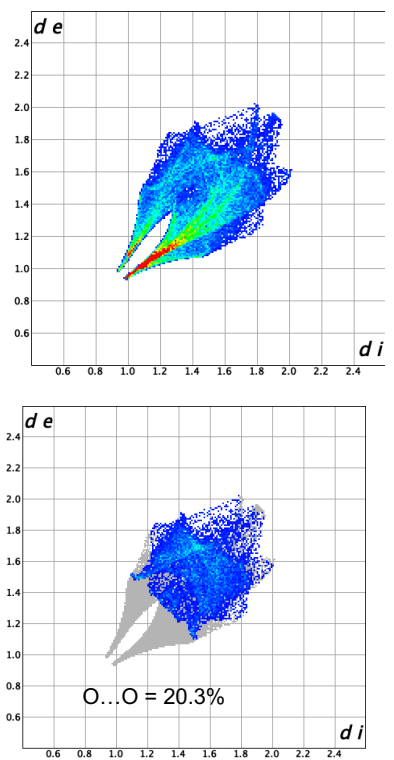

(d)

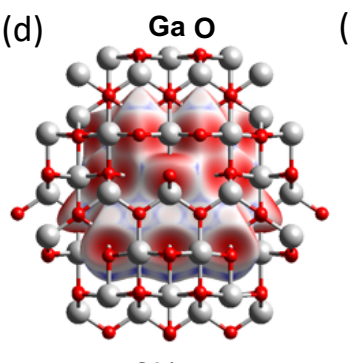

$C 2 / m$

(f)

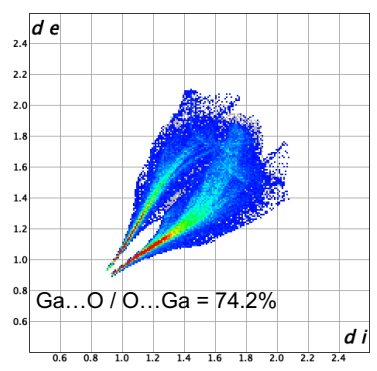

(e)
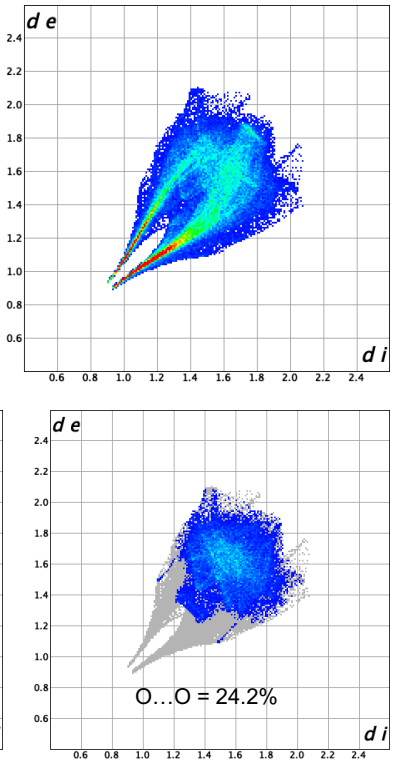

Fig. 3 Hirshfeld surface analysis. (a and d) Hirshfeld surfaces mapped over $d_{\text {norm }}$ for $\alpha$ - and $\beta-\mathrm{Ga}_{2} \mathrm{O}_{3}$ respectively. (b and e) Two dimensional fingerprint plots of $\alpha$ - and $\beta-\mathrm{Ga}_{2} \mathrm{O}_{3}$ respectively. (c and f) Fingerprint plots delineated into $\mathrm{Ga} \cdots \mathrm{O} / \mathrm{O} \cdots \mathrm{Ga}$ and $\mathrm{O} \cdots \mathrm{O}$ contacts for $\alpha-$ and $\beta-\mathrm{Ga}_{2} \mathrm{O}_{3}$ respectively.

The thermostability of the $\mathrm{Ga}_{2} \mathrm{O}_{3}$ sheets was explored using thermogravimetric analysis (TGA) and differential scanning calorimetry (DSC). The TGA (Fig. 2e blue line) shows a small weight loss between 90-100 ${ }^{0} \mathrm{C}$ associated with loss of the solvent $n$-propanol physisorbed on the surface of the sheets. A slight weight increase at $\sim 495{ }^{\circ} \mathrm{C}$ which we predict corresponds to gallium metal and/or subvalent gallium present sandwiched within the sheets. The DSC (Fig. 2e red line) shows a thermodynamic response associated with a phase transition at $\sim 875{ }^{\circ} \mathrm{C}$ which coincides with a weight increase. SEM images show that the phase transition was due to a change in morphology of the ultrathin sheets to fibres of $\mathrm{Ga}_{2} \mathrm{O}_{3}$ which were shown to be in the $\beta-\mathrm{Ga}_{2} \mathrm{O}_{3}$ phase (Supplementary Fig. S6 and Fig. S7). 


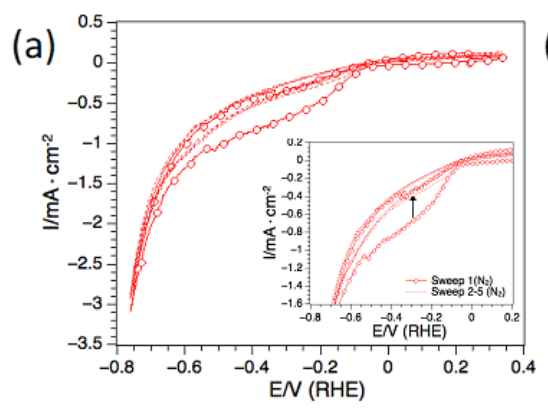

(d)

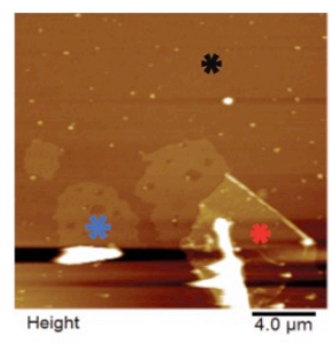

(f)
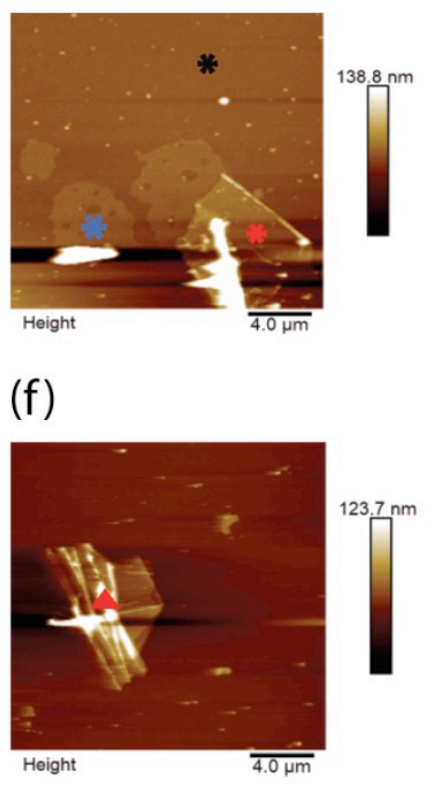
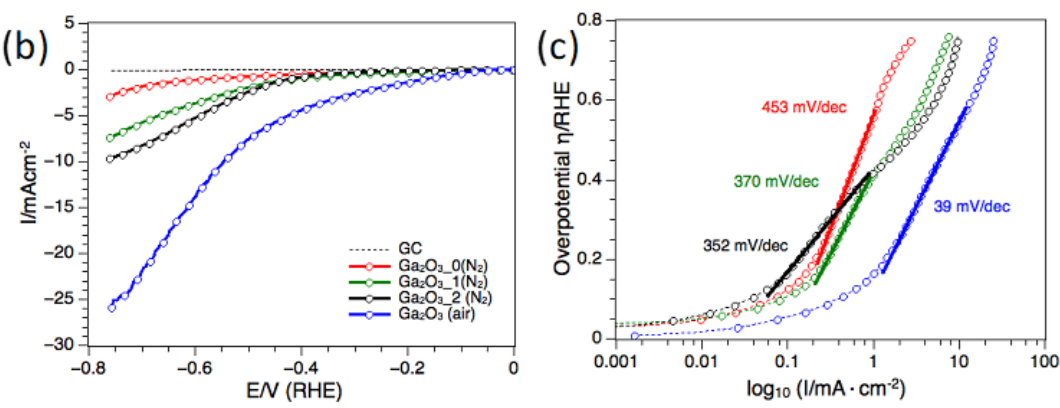

(e)

(g)
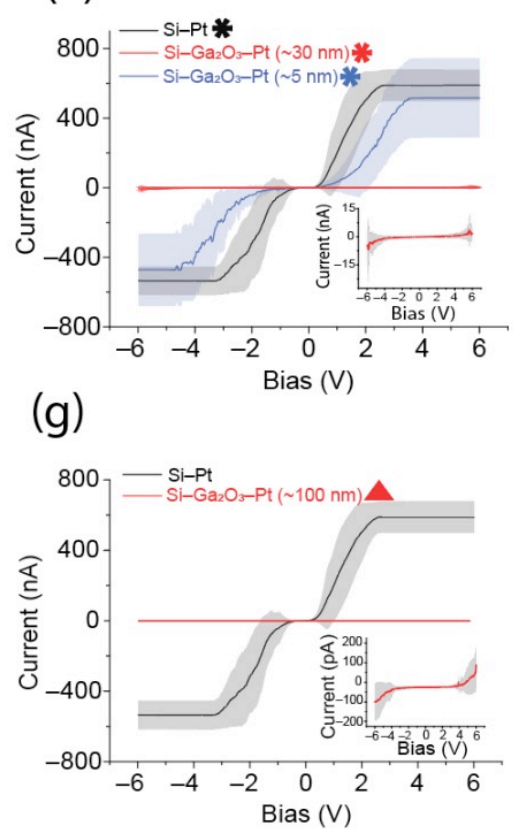

Fig. 4 Applications of the ultrathin $\mathrm{Ga}_{2} \mathrm{O}_{3}$ sheets. (a-c) Electrocatalytic hydrogen evolution reaction of $\mathbf{G a}_{2} \mathrm{O}_{3}$ in $0.5 \mathrm{M} \mathrm{H}_{2} \mathrm{SO}_{4}$; a Cyclic voltammogram traces of $70.4 \mu \mathrm{g}^{-1} \bullet \mathrm{cm}^{-2}$ as-prepared $\mathrm{Ga}_{2} \mathrm{O}_{3}$ showing stripping of surface oxygen in the first scan (inset); $\mathbf{b}$ Forward scans of $70.4 \mu \mathrm{g}^{-1} \bullet \mathrm{cm}^{-2}$ as-prepared $\mathrm{Ga}_{2} \mathrm{O}_{3} \mathrm{NS}$ under $\mathrm{N}_{2}$ (red), after first (green) and second (black) conditioning cycle between -0.8 and $1.8 \mathrm{~V}$ vs. RHE. The blue $\mathrm{CV}$ trace represents HER of $140.8 \mathrm{mg}^{-1} \mathrm{~cm}^{-2} \mathrm{Ga}_{2} \mathrm{O}_{3}$ under atmospheric gas (air); $\mathbf{c}$ respective Tafel slopes under different gas conditions and mass-loadings. The low Tafel slope $\left(39 \mathrm{mV}^{-1} \mathrm{dec}^{-1}\right)$ and low overpotential $\left(\eta\left(1 \mathrm{~mA} \bullet \mathrm{cm}^{-2}\right)=150 \mathrm{mV}\right.$ vs. RHE) of $\mathrm{Ga}_{2} \mathrm{O}_{3}$ in this study exhibits activities toward HER which are comparable to platinum group metals on carbon support [51], transition metal phosphides [52] and hybrid metal nanoparticles [53], d-e Current-potential relationship of the $\mathrm{Ga}_{2} \mathrm{O}_{3}$ sheets compared to silicon; $\mathbf{d}$ AFM topography image of gallium oxide sheets with thickness of 5 to $30 \mathrm{~nm}$; e I-V measurement compered between $\mathrm{Si}_{-}-\mathrm{Ga}_{2} \mathrm{O}_{3}-\mathrm{Pt}(5 \mathrm{~nm}$ (blue) and $30 \mathrm{~nm}$ (red)), and $\mathrm{Si}-\mathrm{Pt}$ (black) junctions. Inset in e corresponds to 30 $\mathrm{nm} \mathrm{Ga}_{2} \mathrm{O}_{3}$ sheet's I-V curve, in scale of $n A$ for current; $\mathbf{f}$ AFM topography image of $\mathrm{Ga}_{2} \mathrm{O}_{3}$ wrinkled area with $\sim 100 \mathrm{~nm}$ thickness; $\mathrm{g}$ I-V measurement compered between $\mathrm{Si}-\mathrm{Ga}_{2} \mathrm{O}_{3}-\mathrm{Pt}(\sim 100 \mathrm{~nm}$ (red)) and $\mathrm{Si}-\mathrm{Pt}$ (black) junctions. Inset in $\mathrm{g}$ corresponds to $100 \mathrm{~nm} \mathrm{Ga}_{2} \mathrm{O}_{3}$ sheet's $\mathrm{I}-\mathrm{V}$ curve, in scale of $\mathrm{pA}$ for current. The bias is applied to the surface with bias sweep set between -6 to $6 \mathrm{~V}$. ${ }^{*}, \boldsymbol{\Lambda}$, are the location where the IV curves were 
collected. Statistics were obtained by measuring the IVs at different locations around the marks in an area of $1 \mu \mathrm{m}^{2}$. Lines are the average of 50 curves collected from each location.

$\mathrm{Ga}_{2} \mathrm{O}_{3}$ as an active catalyst in electrocatalytic hydrogen evolution reactions (HER). $\mathrm{Ga}_{2} \mathrm{O}_{3}$ nanostructures have been shown to be efficient supports in photocatalytic water splitting and $\mathrm{CO}_{2}$ reduction [54-58]. However, little is known about the electrocatalytic hydrogen evolution reaction (HER) [59]. Herein we report the catalytic activity of the $\mathrm{Ga}_{2} \mathrm{O}_{3}$ nanosheets toward electrochemical HER in $0.5 \mathrm{M} \mathrm{H}_{2} \mathrm{SO}_{4}$. The recorded cyclic voltammogram traces and calculated Tafel slopes are shown in Fig. 4a-c. The as prepared $\mathrm{Ga}_{2} \mathrm{O}_{3}$ nanostructures on the glassy carbon (GC) electrode revealed under protective gas $\left(\mathrm{N}_{2}\right)$ a broad reduction event between -0.1 and -0.6 volts vs. RHE during the first cyclic voltammetry scan, which disappears during the subsequent numbers of sweeps (Fig. 4a, inset). The broad cathodic reduction during the first scan was reproduced when the same nanostructures were conditioned between -0.8 and 1.8 volts vs. RHE (Supplementary Fig. S8) and studied again under cathodic potentials. Since no dissolved oxygen was present, the first scan event is associated with stripping of the oxide layer that is regenerated under anodic potentials. The oxide layer remains stable during the subsequent conditioning cycles suggesting that the gallium nanosheets react with water under oxidative potentials (Supplementary Fig. S8). Further studies under oxygen-free and ambient conditions showed that the oxide layer plays a crucial role during the electrocatalytic activity of HER. When replacing the nitrogen by atmospheric gas (air) and increasing the mass loading, the $\mathrm{Ga}_{2} \mathrm{O}_{3}$ nanosheets achieved the highest activity toward HER (Fig. 4b). The calculated Tafel slope of $39 \mathrm{mVdec}^{-1}$ (Fig. 4c) over a wide range of overpotential, $150-600 \mathrm{mV}$ vs. RHE, reveals high production rates of $\mathrm{H}_{2}$ and suggests that Heyrovsky and Tafel determining rate steps are involved in the mechanism [60]. This is likely due to the absorbed protons by surface oxygen since the highest hydrogen production rate appears also in the potential range where stripping of the oxygen layer occurs (Fig. 4a). The role of surface oxygen as active sites to promote the hydrogen evolution reaction has also been shown in photocatalytic water splitting [58].

Studying the electronic properties of single $\mathrm{Ga}_{2} \mathrm{O}_{3}$ sheets. Functional oxides, mainly $\mathrm{SiO}_{2}$, are fundamental to modern microelectronics as high quality insulators, electroluminescent and electrochromic materials, amongst other applications. Considerable effort over several decades has resulted in high quality insulating materials now ubiquitously used in complementary metal oxide semiconductor devices. To really push these technologies into the future, the next-generation of electronic components that can handle great power loads without increasing the size of the electronics systems is needed. Modern devices incorporate few-nanometer thick layers in which electrical stress can be extreme. Therefore, in recent years much effort has been devoted to develop novel gate dielectrics as alternative to silicon oxide for miniaturised electronics. Among them, $\mathrm{Ga}_{2} \mathrm{O}_{3}$ is one of the most promising dielectric materials due to its wide bandgap $(\sim 4.8 \mathrm{eV})$ and excellent chemical/thermal stability. Here, we have studied the insulating electrical properties of the fabricated $\mathrm{Ga}_{2} \mathrm{O}_{3}$ sheets via conducting atomic force microscopy by wiring $\mathrm{Ga}_{2} \mathrm{O}_{3}$ sheets of different thicknesses. A highly doped silicon surface was used as the base electrode and a platinum electrode as the tip electrode and the fabricated thin layers of $\mathrm{Ga}_{2} \mathrm{O}_{3}$ in between. Results show that the current is reduced by over three orders of magnitude (Fig. 4 d-g) when the thickness of the $\mathrm{Ga}_{2} \mathrm{O}_{3}$ layer reaches $100 \mathrm{~nm}$ (Supplementary Table S1). Currents 
comparable to a bare Pt-Si junction are only observed above $6 \mathrm{~V}$ for a $\mathrm{Ga}_{2} \mathrm{O}_{3}$ layer of $30 \mathrm{~nm}$ thick (Fig. 4 inset in e). This translates to a breakdown voltage of $>0.2 \mathrm{~V} / \mathrm{nm}$ for the fabricated $\mathrm{Ga}_{2} \mathrm{O}_{3}$ layers which is comparable to highly insulating materials such as $\mathrm{SiOx}(\sim 0.4 \mathrm{~V} / \mathrm{nm})$ that are commonly used as the insulating gate electrodes in metal-oxide-semiconductor field-effect transistor [61, 62]. This is also consistent with recent studies demonstrating $\mathrm{Ga}_{2} \mathrm{O}_{3}$ as an effective gate electrode in transistors [63-65].

\section{Conclusions}

We have established a significant advancement in the area of 2D-nanomaterials, with the development of a simple and robust method for the exfoliation of ultrathin $\mathrm{Ga}_{2} \mathrm{O}_{3}$ nanosheets with an average thickness of $\sim 5-6$ $\mathrm{nm}$, directly from gallium metal. The simple and benign method generates the $2 \mathrm{D}$ material in $\sim 60 \%$ yield, which is dramatically higher than has been achieved using other methods, and it avoids the use of high molecular weight solvents, surfactants and chemical stabilisers. We have also ensure scalability of the process is addressed at the inception of the science for industry uptake, with the processing under continuous flow. We envisage that this method can be extended to other low melting point metals and metalloids, for fabricating analogous layered and non-layered nanomaterials. The ability to exfoliate the oxide layer from gallium metal suggests that there must be some tangential force associated with lift mechanism in overcoming the centrifugal force in the rotating tube which would otherwise pin the high density oxide to the surface of thin film of gallium metal. Both the tangential force and lift mechanism is consistent with the presence of micron/sub-micron spinning top topological fluid flow in the VFD. This is one of the topological fluid flows in the general model of fluid flow in the VFD which accounts for all the processing outcomes of the device, depending on the nature of the liquid and rotational speed, at a tilt angle of $45^{\circ}$ [46] In addition, the ability to prepare highly insulating gallium oxide sheets has potential in device technology, and the catalytic prowess of the ultrathin sheets shows promise for application in water splitting in avoiding the use of precious metals of the transition metals and material for which its use as such is questionable on environmental grounds.

\section{References}

1. Kalantar-Zadeh, K. et al. Emergence of Liquid Metals in Nanotechnology. ACS Nano 13, 7388-7395 (2019).

2. Daeneke, T. et al. Liquid metals: fundamentals and applications in chemistry. Chemical Society Reviews 47, 4073-4111 (2018).

3. Messalea, K.A. et al. $\mathrm{Bi}_{2} \mathrm{O}_{3}$ monolayers from elemental liquid bismuth. Nanoscale 10, 15615-15623 (2018).

4. Novoselov, K.S. et al. Electric Field Effect in Atomically Thin Carbon Films. Science 306, 666 (2004).

5. Novoselov, K.S. et al. Two-dimensional atomic crystals. Proceedings of the National Academy of Sciences of the United States of America 102, 10451 (2005).

6. Coleman, J.N. et al., Two-Dimensional Nanosheets Produced by Liquid Exfoliation of Layered Materials. Science 331, 568 (2011).

7. Nicolosi, V. et al. Liquid Exfoliation of Layered Materials. Science 340, 1226419 (2013). 
8. Hanlon, D. et al. Liquid exfoliation of solvent-stabilized few-layer black phosphorus for applications beyond electronics. Nature Communications 6, 8563 (2015).

9. Lee, C. et al. Anomalous Lattice Vibrations of Single- and Few-Layer MoS 2 . ACS Nano 4, 2695-2700 (2010).

10. Liu, K. K. et al. Growth of large-area and highly crystalline $\mathrm{MoS}_{2}$ thin layers on insulating substrates. Nano Lett 12, 1538-44 (2012).

11. Peng, J. et al. Two-Dimensional Tellurium Nanosheets Exhibiting an Anomalous Switchable Photoresponse with Thickness Dependence. Angewandte Chemie International Edition 57, 1353313537 (2018).

12. Wang, Q. and O'Hare, D. Recent Advances in the Synthesis and Application of Layered Double Hydroxide (LDH) Nanosheets. Chemical Reviews 112, 4124-4155 (2012).

13. Smith, R.J. et al. Large-Scale Exfoliation of Inorganic Layered Compounds in Aqueous Surfactant Solutions. Advanced Materials 23, 3944-3948 (2011).

14. Sachdev, H. Disclosing boron's thinnest side. Science, 350, 1468 (2015).

15. Bianco, E. et al. Stability and Exfoliation of Germanane: A Germanium Graphane Analogue. ACS Nano 7, 4414-4421 (2013).

16. Guo, X. et al. Room-temperature liquid metal and alloy systems for energy storage applications. Energy \& Environmental Science 12, 2605-2619 (2019).

17. Iqbal, N. et al. Sonochemical Assisted Solvothermal Synthesis of Gallium Oxynitride Nanosheets and their Solar-Driven Photoelectrochemical Water-Splitting Applications. Scientific Reports 6, 32319 (2016).

18. Alsaif, M.M.Y.A. et al. Atomically Thin $\mathrm{Ga}_{2} \mathrm{~S}_{3}$ from Skin of Liquid Metals for Electrical, Optical, and Sensing Applications. ACS Applied Nano Materials 2, 4665-4672 (2019).

19. Dickey, M.D. Stretchable and Soft Electronics using Liquid Metals. Advanced Materials 29, 1606425 (2017).

20. Carey, B.J. et al. Wafer-scale two-dimensional semiconductors from printed oxide skin of liquid metals. Nature Communication 8, 14482 (2017).

21. Zhang, X. et al. Phase Transition of Two-Dimensional $\beta-\mathrm{Ga} 2 \mathrm{O} 3$ Nanosheets from Ultrathin $\gamma-\mathrm{Ga}_{2} \mathrm{O}_{3}$ Nanosheets and Their Photocatalytic Hydrogen Evolution Activities. ACS Omega 3, 14469-14476 (2018).

22. Kochat, V. et al. Atomically thin gallium layers from solid-melt exfoliation. Science Advances 4, 1701373 (2018).

23. Syed, N. et al. Printing two-dimensional gallium phosphate out of liquid metal. Nature Communications 9, 3618 (2018).

24. Jones, D.B. et al. Plasma enhanced vortex fluidic device manipulation of graphene oxide. Chemical Communications 52, 10755-10758 (2016).

25. Al-Antaki, A.H.M. et al. Inverted vortex fluidic exfoliation and scrolling of hexagonal-boron nitride. RSC Advances 9, 22074-22079 (2019). 
26. Batmunkh, M. et al. Efficient Production of Phosphorene Nanosheets via Shear Stress Mediated Exfoliation for Low-Temperature Perovskite Solar Cells. Small Methods 3, 1800521 (2019).

27. Vimalanathan, K. et al. Fluid dynamic lateral slicing of high tensile strength carbon nanotubes. Scientific Reports 6, 22865 (2016).

28. Alharbi, T.M.D. et al. Controlled slicing of single walled carbon nanotubes under continuous flow. Carbon 140, 428-432 (2018).

29. Britton, J. et al. Vortex Fluidic Chemical Transformations. Chemistry - A European Journal 23, 13270-13278 (2017).

30. Totoiu, C.A. et al. Vortex fluidics-mediated DNA rescue from formalin-fixed museum specimens. PLoS ONE 2695-2700 (2020).

31. Phillips, J.M. et al. Chemoselective and Continuous Flow Hydrogenations in Thin Films Using a Palladium Nanoparticle Catalyst Embedded in Cellulose Paper. ACS Applied Bio Materials 2, 488494 (2019).

32. Luo, X. et al. Laser irradiated vortex fluidic mediated synthesis of luminescent carbon nanodots under continuous flow. Reaction Chemistry \& Engineering 3, 164-170 (2018).

33. Pye, S.J. et al. Organic oxidations promoted in vortex driven thin films under continuous flow. Green Chemistry 20, 118-124 (2018).

34. Heine, V. Crystal structure of gallium metal. Journal of Physics C: Solid State Physics 1, 222-231 (1968).

35. Robinson, G.H. Gallanes, Gallenes, Cyclogallenes, and Gallynes: Organometallic Chemistry about the Gallium-Gallium Bond. Accounts of Chemical Research 32, 773-782 (1999).

36. Ghigna, P. et al. Metallic versus covalent bonding: Ga nanoparticles as a case study. J Am Chem Soc 129, 8026-8033 (2007).

37. Gao, Y. and Bando Y. Carbon nanothermometer containing gallium. Nature, 415, 599-599 (2002).

38. Qin, B. and Schneider U. Catalytic Use of Elemental Gallium for Carbon-Carbon Bond Formation. Journal of the American Chemical Society 138, 13119-13122 (2016).

39. Spells, K.E. The determination of the viscosity of liquid gallium over an extended nrange of temperature. Proceedings of the Physical Society 48, 299-311 (1936).

40. Zavabeti, A. et al. A liquid metal reaction environment for the room-temperature synthesis of atomically thin metal oxides. Science 358, 332 (2017).

41. Syed, N. et al. Sonication-Assisted Synthesis of Gallium Oxide Suspensions Featuring Trap State Absorption: Test of Photochemistry. Advanced Functional Materials 27, 1702295 (2017).

42. Tang, S.-Y. et al. Functional Liquid Metal Nanoparticles Produced by Liquid-Based Nebulization. Advanced Materials Technologies 4, 1800420 (2019).

43. Vimalanathan, K., Chen, X. and Raston C.L. Shear induced fabrication of intertwined single walled carbon nanotube rings. Chemical Communications 50, 11295-11298 (2014).

44. Vimalanathan, K. et al. Surfactant-free Fabrication of Fullerene $\mathrm{C}_{60}$ Nanotubules Under Shear. Angewandte Chemie International Edition 56, 8398-8401 (2017). 
45. Luo, X. et al. Vortex Fluidic Device-Intensified Aqueous Two Phase Extraction of C-Phycocyanin from Spirulina Maxima. ACS Sustainable Chemistry \& Engineering 4, 3905-3911 (2016).

46. Thaar M. D. A. et al. Sub-Micron Moulding Topological Mass Transport Regimes in Angled Vortex Fluidic Flow. Nanoscale Adv. DOI: 10.1039/d1na00195g (2021).

47. Regan, M.J. et al. X-ray study of the oxidation of liquid-gallium surfaces. Physical Review B 55, 10786-10790 (1997).

48. Creighton, J.A. and Withnall R. The Raman spectrum of gallium metal. Chemical Physics Letters 326, 311-313 (2000).

49. Wundrack, S. et al. Graphene meets gallenene -- A straightforward approach to developing large-area heterostacks by gallium self-propagation. arXiv: Materials Science (2019).

50. Bosi, M. et al. $\mathrm{Ga}_{2} \mathrm{O}_{3}$ polymorphs: tailoring the epitaxial growth conditions. Journal of Materials Chemistry C 8, 10975-10992 (2020).

51. Ponomarenko, L.A. et al. Cloning of Dirac fermions in graphene superlattices. Nature, 497, 594-597 (2013).

52. Yang, H. et al. Dielectric nanosheets made by liquid-phase exfoliation in water and their use in graphene-based electronics. 2D Materials 1, 011012 (2014).

53. Joishi, C., et al., Breakdown Characteristics of $\beta-(\mathrm{Al} 10.22 \mathrm{Ga} 0.78)_{2} \mathrm{O}_{3} / \mathrm{Ga}_{2} \mathrm{O}_{3}$ Field-Plated ModulationDoped Field-Effect Transistors. IEEE Electron Device Letters, 40, 1241-1244 (2019).

54. Kamimura, T., Nakata, Y. and Higashiwaki, M. Effect of $(\mathrm{AlGa})_{2} \mathrm{O}_{3}$ back barrier on device characteristics of $\beta-\mathrm{Ga}_{2} \mathrm{O}_{3}$ metal-oxide-semiconductor field-effect transistors with Si-implanted channel. Japanese Journal of Applied Physics 60, 030906 (2021).

55. Wong, M.H. et al. Current Aperture Vertical $\beta-\mathrm{Ga}_{2} \mathrm{O}_{3}$ MOSFETs Fabricated by N- and Si-Ion Implantation Doping. IEEE Electron Device Letters 40, 431-434 (2019).

56. Yang, J. et al. Efficient and Robust Hydrogen Evolution: Phosphorus Nitride Imide Nanotubes as Supports for Anchoring Single Ruthenium Sites. Angewandte Chemie International Edition 57, 94959500 (2018).

57. Wang, D. et al. Influence of Mo/P Ratio on CoMoP nanoparticles as highly efficient HER catalysts. Applied Catalysis A: General 511, 11-15 (2016).

58. Amin, M.A. et al. Aluminum Titania Nanoparticle Composites as Nonprecious Catalysts for Efficient Electrochemical Generation of $\mathrm{H}_{2}$. ACS Applied Materials \& Interfaces 8, 23655-23667 (2016).

59. Busser, G.W. et al. Cocatalyst Designing: A Regenerable Molybdenum-Containing Ternary Cocatalyst System for Efficient Photocatalytic Water Splitting. ACS Catalysis 5, 5530-5539 (2015).

60. Yanagida, T., Sakata, Y. and Imamura, H. Photocatalytic Decomposition of $\mathrm{H}_{2} \mathrm{O}$ into $\mathrm{H}_{2}$ and $\mathrm{O}_{2}$ over $\mathrm{Ga}_{2} \mathrm{O}_{3}$ Loaded with NiO. Chemistry Letters 33, 726-727 (2004).

61. Wang, X. et al. Photocatalytic Overall Water Splitting Promoted by an $\alpha-\beta$ phase Junction on $\mathrm{Ga}_{2} \mathrm{O}_{3}$. Angewandte Chemie International Edition 51, 13089-13092 (2012).

62. Yamamoto, M. et al. Photocatalytic reduction of $\mathrm{CO}_{2}$ with water promoted by $\mathrm{Ag}$ clusters in $\mathrm{Ag} / \mathrm{Ga}_{2} \mathrm{O}_{3}$ photocatalysts. Journal of Materials Chemistry A 3, 16810-16816 (2015). 
63. Wang, Y. et al. Unraveling the Mechanism of Photocatalytic Water Splitting in $\alpha-\mathrm{Ga}_{2} \mathrm{O}_{3}$ Loaded with a Nickel Oxide Cocatalyst: A First-Principles Investigation. The Journal of Physical Chemistry C 123, 8990-9000 (2019).

64. Kakoria, A. et al. Gallium Oxide Nanofibers for Hydrogen Evolution and Oxygen Reduction. ACS Applied Nano Materials 2, 64-74 (2019).

65. Shinagawa, T., Garcia-Esparza, A.T. and Takanabe, K. Insight on Tafel Slopes from a Microkinetic Analysis of Aqueous Electrocatalysis for Energy Conversion. Scientific Reports 5, 13801 (2015).

Supplementary information is linked to the online version of the paper at www.nature.com/nature.

Acknowledgements Support of this work by the Australian Research Council and the Government of South Australia is greatly acknowledged. The authors also acknowledge the helpful discussions with Professor Allan Pring (Flinders University) and the expertise, equipment and support provided by Microscopy Australia (MA) and the Australian National Fabrication Facility (ANFF) and the South Australian Nodes under the National Collaborative Research Infrastructure Strategy.

Author contributions K.V. and C.L.R. designed the experiments and wrote the manuscript. T.P performed and optimised the experimental conditions, K.V performed and analyzed the SEM, AFM, Raman, XPS, XRD and TGA/DSC data, Z.G performed the Scanning Auger measurements, I.L. performed the Hirshfeld analysis, S.R. and N.D performed the single particle electronic studies using conductive force microscopy, S.E. performed the hydrogen evolution reactions, J.R.G. performed the SEM/EDX measurements, C.G. performed AFM height analysis, Q.S. and J.Z. performed the TEM and HRTEM measurements and M.R.A. contributed towards the development of experiments related to the hydrogen evolution reactions. All authors contributed towards editing of the manuscript.

Competing interests The authors declare no competing interests.

Author information Reprints and permissions is available at www.nature.com/reprints. All authors declare no compelling financial interests. Readers are welcomed to comment to the online version of this article at www.nature.com/nature. Correspondence and requests for materials should be addressed to kasturi.vimalanathan@flinders.edu.au and colin.raston@flinders.edu.au 
High stress in situ exfoliation of 2D gallium oxide sheets fro... (7.20 MiB) view on ChemRxiv • download file 


\section{Supplementary information}

High shear in situ exfoliation of 2D gallium oxide sheets from centrifugally derived thin films of liquid gallium

Kasturi Vimalanathan ${ }^{1 *}$, Timotheos Palmer ${ }^{1}$, Zoe Gardner ${ }^{1}$, Irene Ling ${ }^{2}$, Soraya Rahpeima ${ }^{1,3}$, Sait Elmas $^{1}$, Jason R. Gascooke ${ }^{1}$, Christopher T. Gibson ${ }^{1,4}$, Qiang Sun ${ }^{5,6}$, Jin Zou ${ }^{5,6}$, Mats R. Anderson ${ }^{1}$, Nadim Darwish ${ }^{3}$ and Colin L. Raston ${ }^{1 *}$

${ }^{1}$ Flinders Institute for Nanoscale Science and Technology, College of Science and Engineering, Flinders University, Adelaide SA 5001, Australia

${ }^{2}$ School of Science, Monash University Malaysia, Jalan Lagoon Selatan, Bandar Sunway, 47500 Selangor, Malaysia

${ }^{3}$ School of Molecular and Life Sciences, Curtin Institute for Functional Molecule and Interfaces, Curtin University, Bentley, Western Australia 6102, Australia

${ }^{4}$ Flinders Microscopy and Microanalysis, College of Science and Engineering, Flinders University, Bedford Park, SA 5042, Australia

${ }^{5}$ Centre for Microscopy and Microanalysis, The University of Queensland, Brisbane, QLD 4072, Australia

${ }^{6}$ Materials Engineering, The University of Queensland, Brisbane, QLD 4072, Australia.

*Corresponding authors email: kasturi.vimalanathan@flinders.edu.au and colin.raston@flinders.edu.au 


\section{Experimental method}

\section{$\underline{\text { Raman Spectroscopy and Microscopy }}$}

Raman spectra were acquired using a Witec alpha300R Raman microscope at an excitation laser wavelength of $532 \mathrm{~nm}$ with a 100X objective (numerical aperture 0.90). Typical integration times for single Raman spectra were typically $30 \mathrm{~s}$ and averaged from 2 to 3 repetitions. Confocal Raman images were acquired with integrations typically 3 to 5 seconds per pixel. Each pixel in the Raman images represents a Raman spectrum with the number of pixels in a typical Raman image representing hundreds to thousands of spectra. Confocal Raman images are generated by plotting the intensity of a specified region of each Raman spectrum that corresponds to a material, versus the X-Y position of the excitation laser as it scans the sample surface.

\section{$\underline{\text { Atomic Force Microscopy }}$}

AFM images were acquired using a Bruker Multimode 8 AFM with a Nanoscope V controller using tapping mode in air, with all parameters including set-point, scan rate, and feedback gains adjusted to optimize image quality. The AFM probes used were Mikromasch HQ:NSC15 Si probes with a nominal spring constant of $40 \mathrm{~N} / \mathrm{m}$ and a nominal tip diameter of $16 \mathrm{~nm}$. The scanner was calibrated in the $\mathrm{x}, \mathrm{y}$, and $\mathrm{z}$ directions using silicon calibration grids (Bruker model numbers VGRP: $10 \mu \mathrm{m}$ pitch, $180 \mathrm{~nm}$ depth, PG: $1 \mu \mathrm{m}$ pitch, $110 \mathrm{~nm}$ depth). Analysis of AFM images was performed using Nanoscope analysis software version 1.4. Presented AFM topography images were flattened, and the cross sections acquired using the section tool in the Nanoscope analysis software.

\section{Scanning Auger Spectroscopy}

The AES analysis was conducted with a PHI 710 Scanning Auger Nanoprobe. The $\mathrm{Ga}_{2} \mathrm{O}_{3}$ sheets were drop-casted onto silicon wafers which were used for the characterisation. $\mathrm{Ar}^{+}$ions were used in the depth profiling for etching the sheets. The Scanning Auger Nanoprobe parameters were as follows: etching speed, $0.24 \mathrm{~nm} \mathrm{~s}^{-1}$; ion voltage, $2 \mathrm{kV}$; interval; $6 \mathrm{~s}$; depth; $40 \mathrm{~nm}$; and scanning area of $0.19 \mu \mathrm{m}$. Silicon dioxide was as a standard for the etching speed determination. The Scanning Electron Microscopy on the Scanning Auger Nanoprobe used a current intensity of $10 \mathrm{nA}$ and an acceleration voltage of $10 \mathrm{kV}$. Ga $\mathrm{LMM}_{\mathrm{L}}, \mathrm{O}_{\mathrm{KLL}}$ and $\mathrm{Si}_{\mathrm{LVV}}$ Auger lines were used for plotting the depth profiles of each sample.

\section{Conductive Atomic Force Microscopy (C-AFM)}

Conductive- atomic force microscopy (C-AFM) was used for imaging and dc conductivities. Currentvoltage $(\mathrm{I}-\mathrm{V})$ data were collected by a Bruker ICON head with peak force Tuna module at room 
temperature using conductive platinum tip (RMN-25PT300B) having spring constant of 18 N.m-1. The image resolution, scan rate and peak force set point were set to 256 points, $1 \mathrm{~Hz}$ and $50 \mathrm{nN}$, respectively. Here, n-type (phosphorus doped) single-side polished Si wafer with the thickness of $500-550 \mu \mathrm{m}$ and $(111) \pm 0.05^{\circ}$ (with resistivity of $<0.002 \Omega . \mathrm{cm}$ ) was used.

\section{$\underline{\text { Electrocatalytic hydrogen evolution reactions (HER) }}$}

Electrocatalytic hydrogen evolution reactions (HER) were performed on an AUTOLAB potentiostat (Metrohm AG, Switzerland) using a glassy carbon (GC) electrode $\left(0.071 \mathrm{~cm}^{2}\right)$ as working (WE), a platinum wire as counter $(\mathrm{CE})$ and $\mathrm{Ag} \mid \mathrm{AgCl}(1 \mathrm{M} \mathrm{KCl})$ as reference electrode $(\mathrm{RE})$ in $0.5 \mathrm{M} \mathrm{H}_{2} \mathrm{SO}_{4}$ as electrolyte solution. The glassy carbon electrode was polished with $0.3 \mu \mathrm{m}$ Alumina (Micro Polish, IONODE) prior to changing the mass loading. Here, 5 or $10 \mu \mathrm{L}$ of $1 \mathrm{mg} / \mathrm{mL} \mathrm{Ga}_{2} \mathrm{O}_{3}$ nanostructures in n-propanol were drop-casted onto the WE, dried under ambient temperature for $3 \mathrm{~h}$ and tested under oxygen-free and atmospheric gas conditions using $\mathrm{N}_{2}$ as protective gas. This equals to 70.4 and $140.8 \mu \mathrm{g} / \mathrm{cm}^{-2}$ mass loading of $\mathrm{Ga}_{2} \mathrm{O}_{3}$ electrocatalyst. Higher mass-loadings showed leaching of the catalysts from the glassy carbon disc once dispersed in $0.5 \mathrm{M} \mathrm{H}_{2} \mathrm{SO}_{4}$ electrolyte solution. Hence, for the sake of reproducibility and to avoid manipulation of the geometric surface of the GC working electrode, only 1 or 2 droplets of electrocatalyst ( 5 and $10 \mu \mathrm{L}$ ) were drop-casted onto the GC surface. All recorded working potentials vs. $\mathrm{Ag} \mid \mathrm{AgCl}(1 \mathrm{M} \mathrm{KCl})$ were converted into the reversible hydrogen electrode $(\mathrm{RHE})$ according to the equation $\mathrm{E}_{(\mathrm{RHE})}=\mathrm{E}_{(\mathrm{Ag} \mid \mathrm{AgCl})}+0.059 \bullet \mathrm{pH}+\mathrm{E}^{0}(\mathrm{Ag} \mid \mathrm{AgCl})$, where $\mathrm{E}^{0}(\mathrm{Ag} \mid \mathrm{AgCl}$, $1 \mathrm{M} \mathrm{KCl})=0.235 \mathrm{~V}[1]$. 


\section{Supplementary Figure 1}

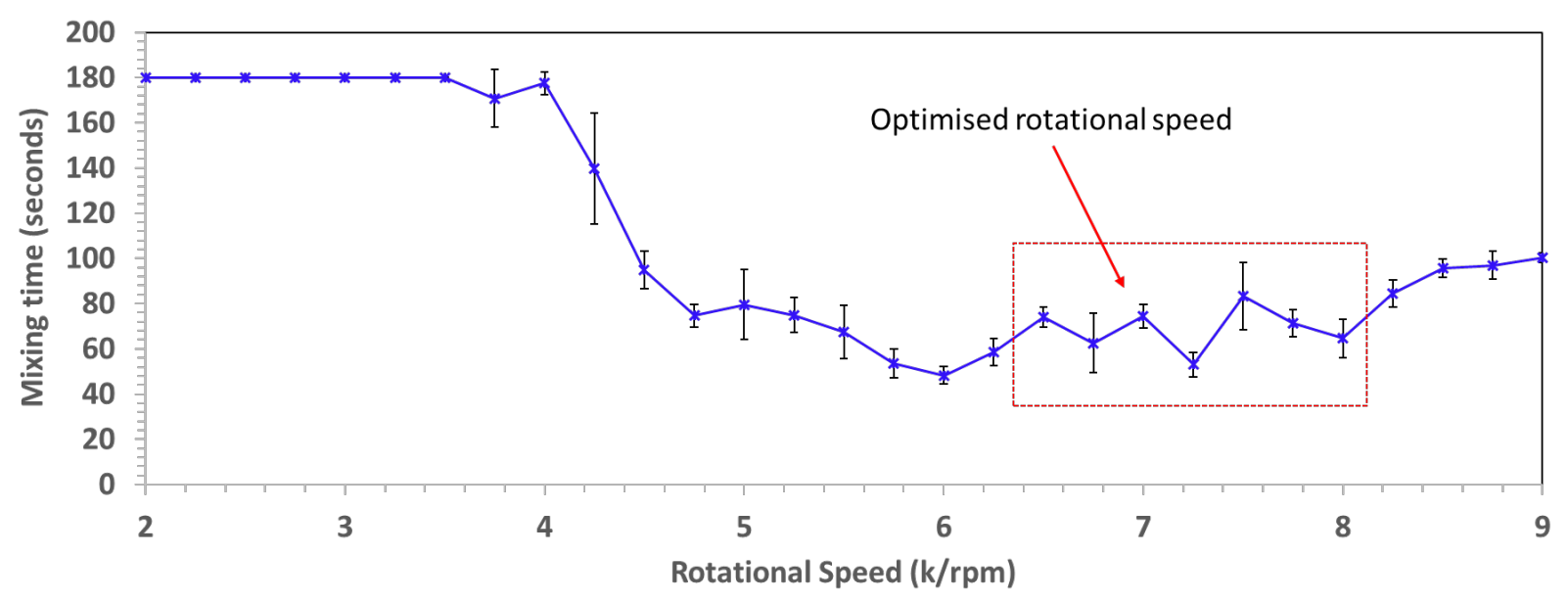

Fig. S1 Mixing times of the solvent in the VFD at the different rotational speeds were measured to identify the optimised speed for the processing of liquid gallium. $1 \mathrm{~mL}$ of $n$-propanol was placed in the VFD operating at an inclination angle of $45^{\circ}$ and varying the rotational speed (2000-9000 rpm) and the time taken for a drop of dye to uniformly mix with the bulk liquid was measured in seconds (all measurements were carried out in triplicates).

\section{Supplementary Figure 2}
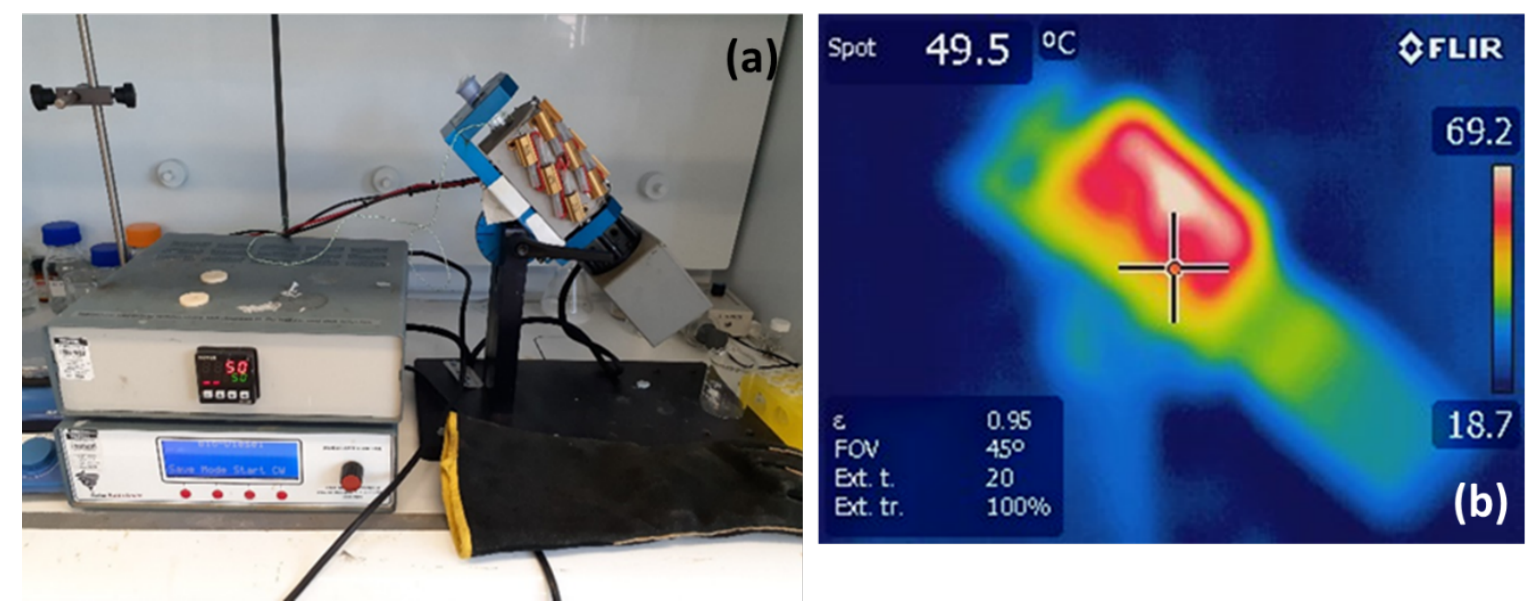

Fig. S2 (a) Control experiment using a 'plug and play' heating jacket as an alternative to heating on a hotplate and (b) Thermal imaging of the VFD with the heating jacked showing a temperature of $50{ }^{0} \mathrm{C}$. 


\section{Supplementary Figure 3}
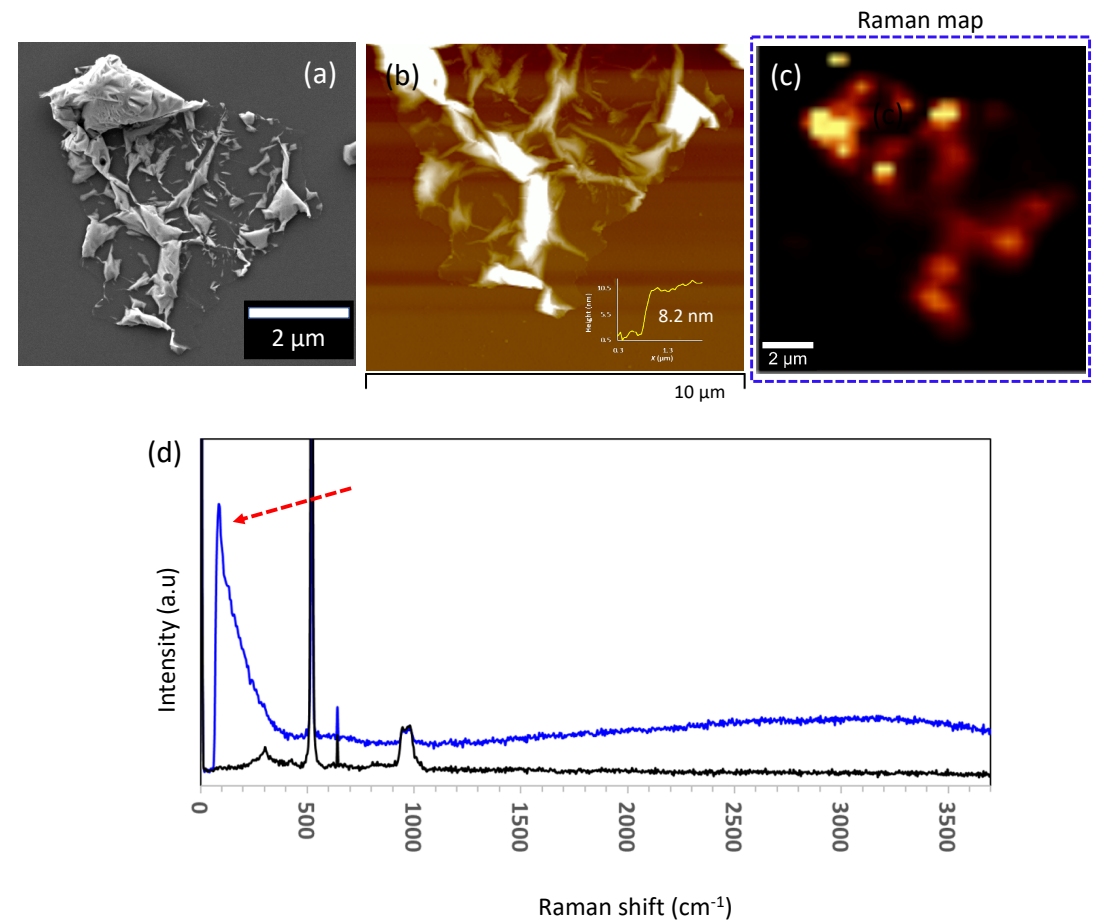

Fig. S3 Raman analysis of a $\mathrm{Ga}_{2} \mathrm{O}_{3}$ sheet; a-b SEM and AFM height image of a $\mathrm{Ga}_{2} \mathrm{O}_{3}$ sheet; $\mathbf{c}$ Raman map of the sheet imaged in (a-b) and $\mathbf{d}$ The Raman spectra that corresponds to the map in (c) mapping the peak labelled with the red arrow, $\sim 50-100 \mathrm{~cm}^{-1}$

\section{Supplementary Figure 4}
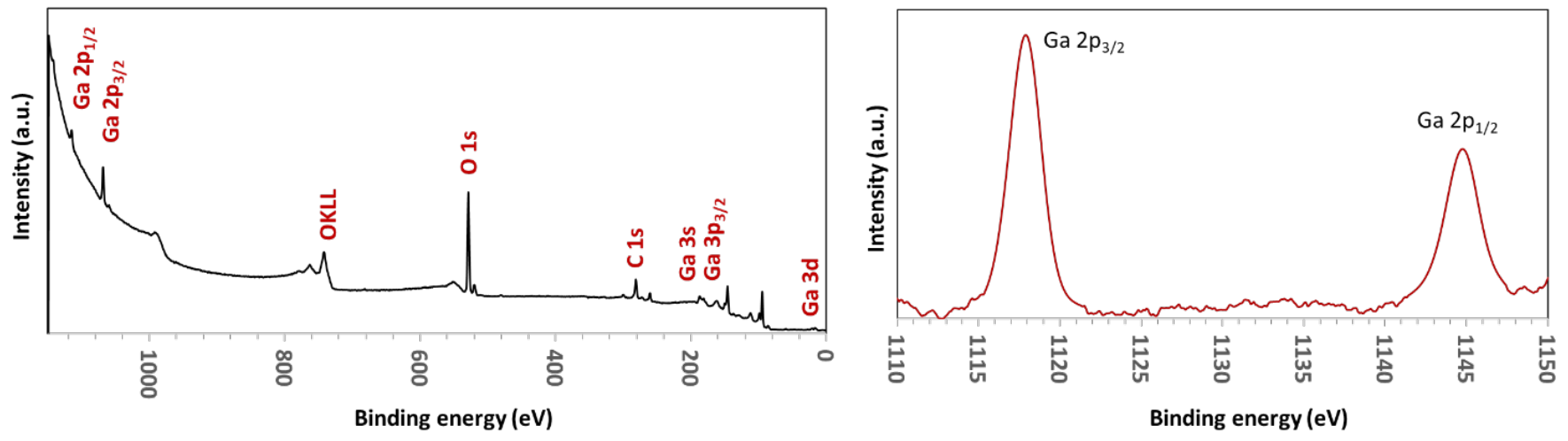

Fig. S4: XPS survey spectra of bulk gallium metal, and (b) High resolution spectra of the doublet $2 \mathrm{p}$ region 


\section{Supplementary Figure 5}
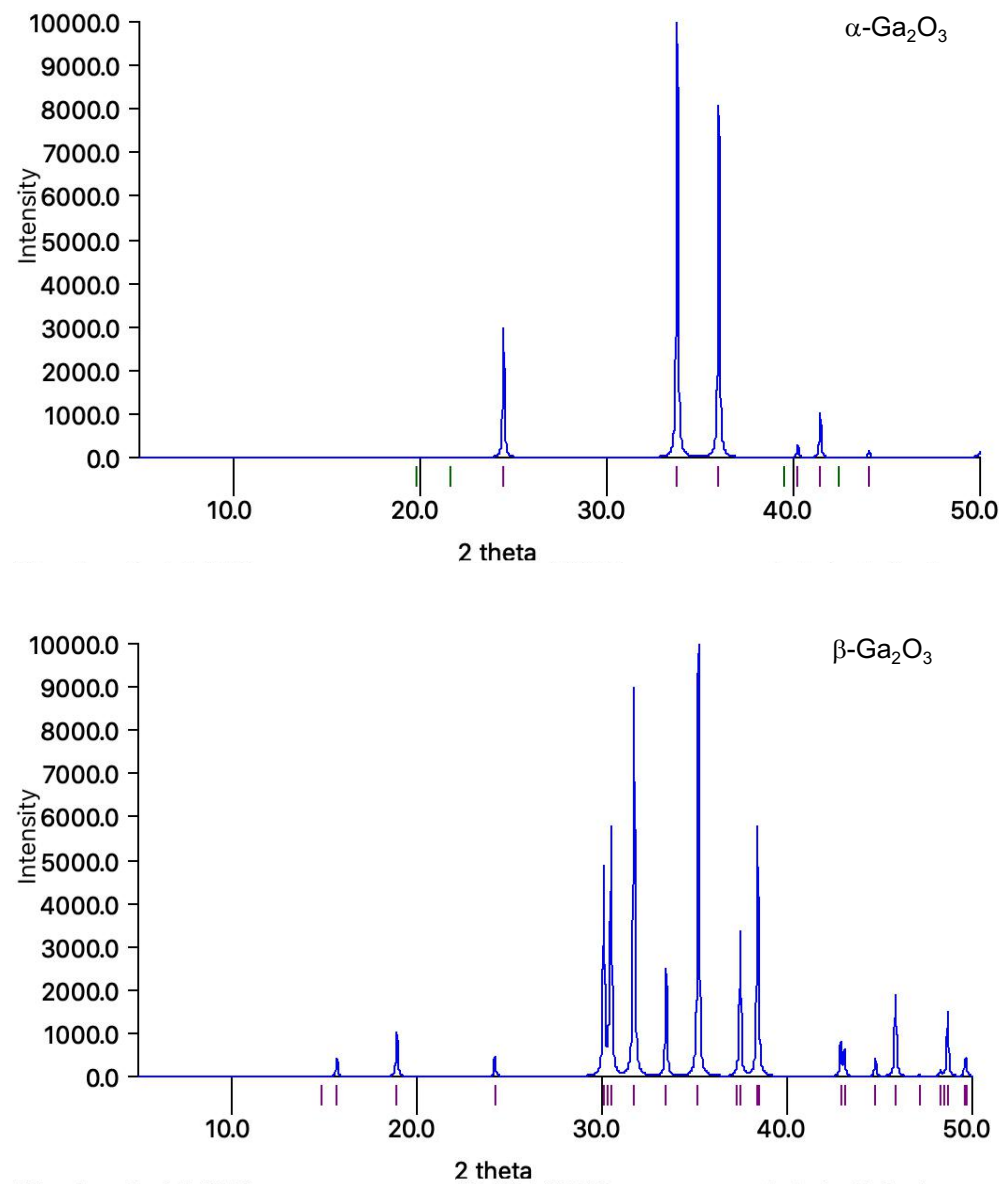

Fig. S5: XRD spectra for $\boldsymbol{\alpha}-$ and $\boldsymbol{\beta}-\mathrm{Ga}_{2} \mathrm{O}_{3}$. Spectra were computed using Mercury software using the crystal structures of the $\alpha$ - and $\beta-\mathrm{Ga}_{2} \mathrm{O}_{3}$ (ICSD numbers: 27431 and 83645 respectively). 


\section{Supplementary Figure 6}

(a)

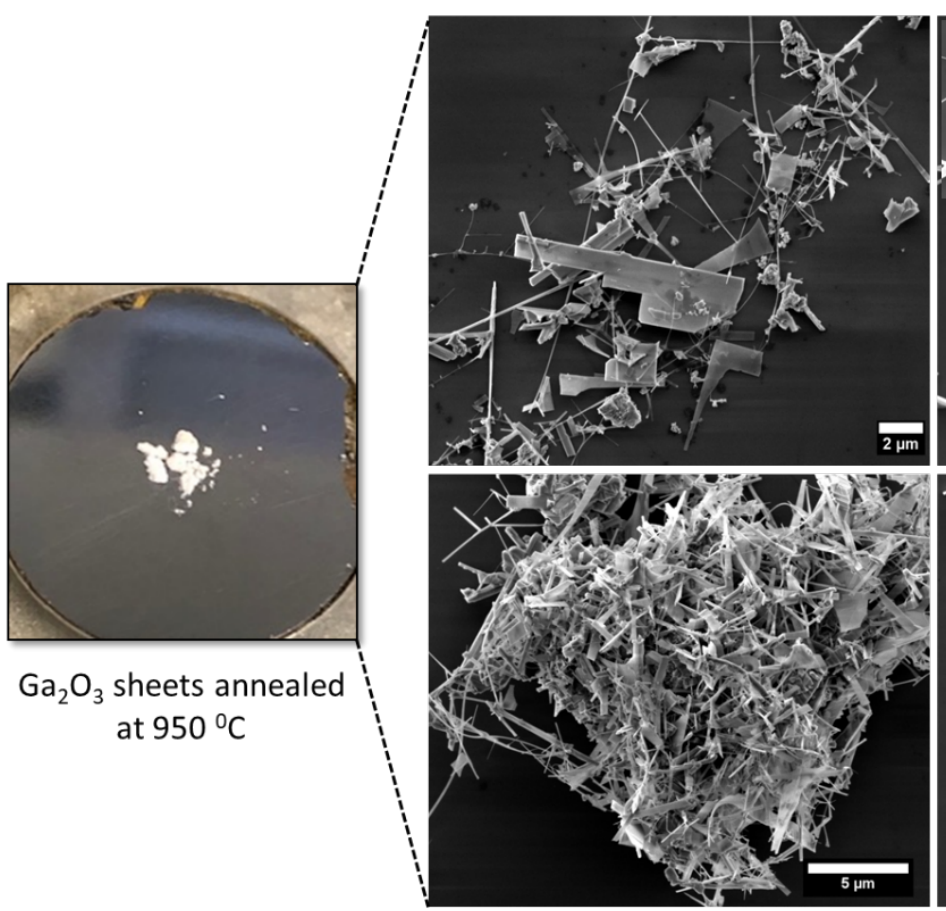

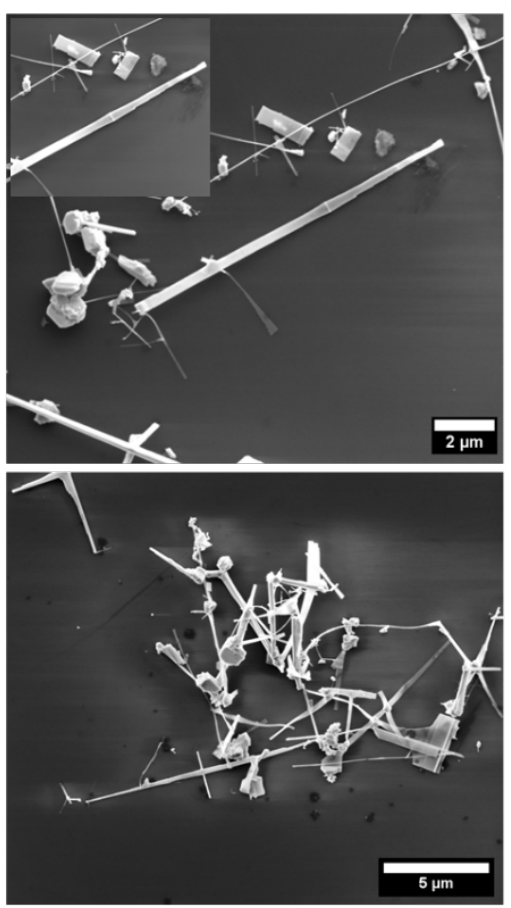

Fig. S6: Characterization of material after thermal analysis. TGA/DSC was carried out at $10{ }^{0} \mathrm{C}$ $\mathrm{min}^{-1}$ of temperature increase under $\mathrm{N}_{2}$. (a) A white powder was collected after heating at $950{ }^{0} \mathrm{C}$ and (b) SEM images of gallium oxide fibres/scrolls formed after heating at $950{ }^{\circ} \mathrm{C}$. 


\section{Supplementary Figure 7}

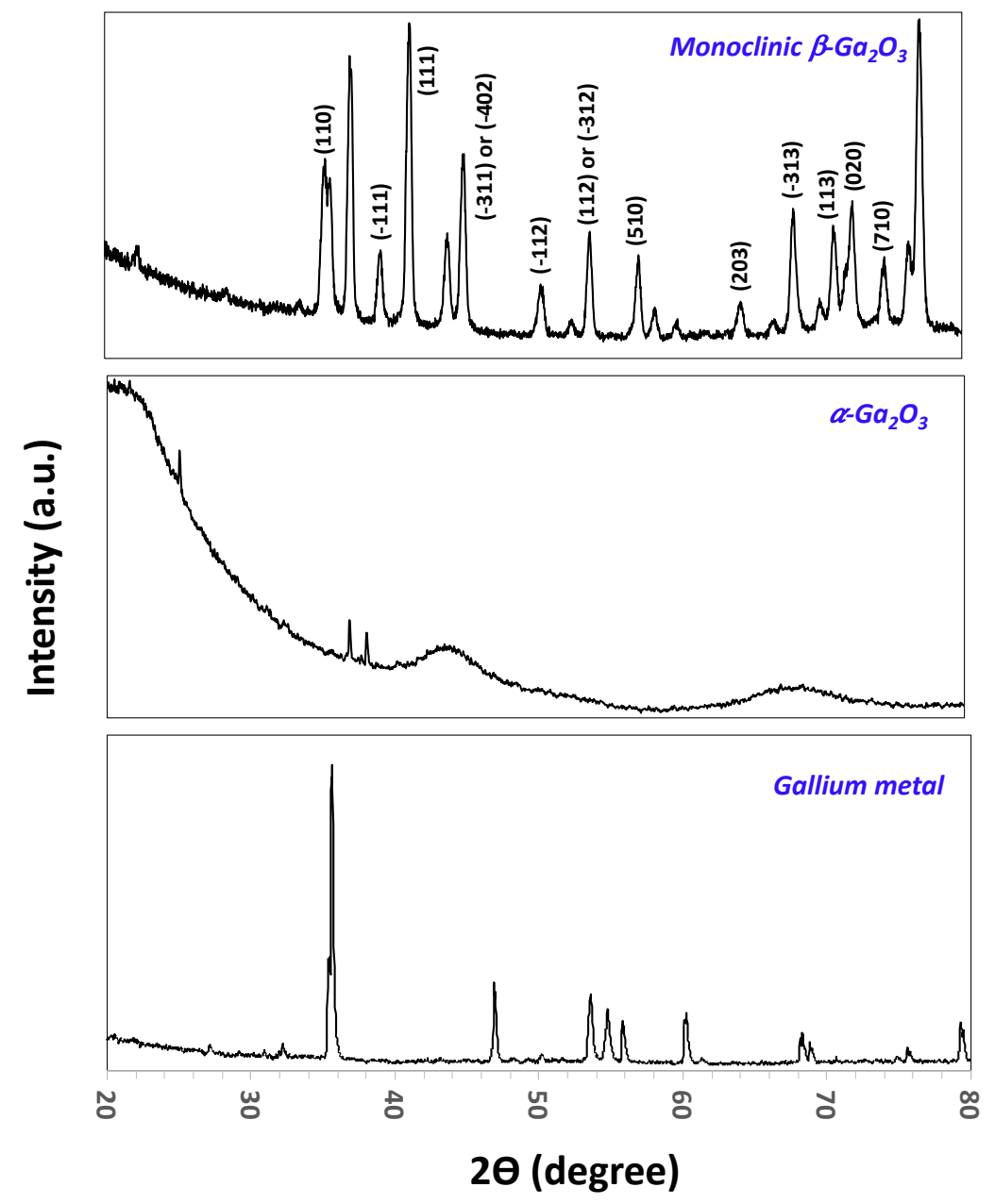

Fig. S7: XRD analysis of the gallium oxide fibres/scrolls formed after heating at $950{ }^{\circ} \mathrm{C}$. 


\section{Supplementary Figure 8}
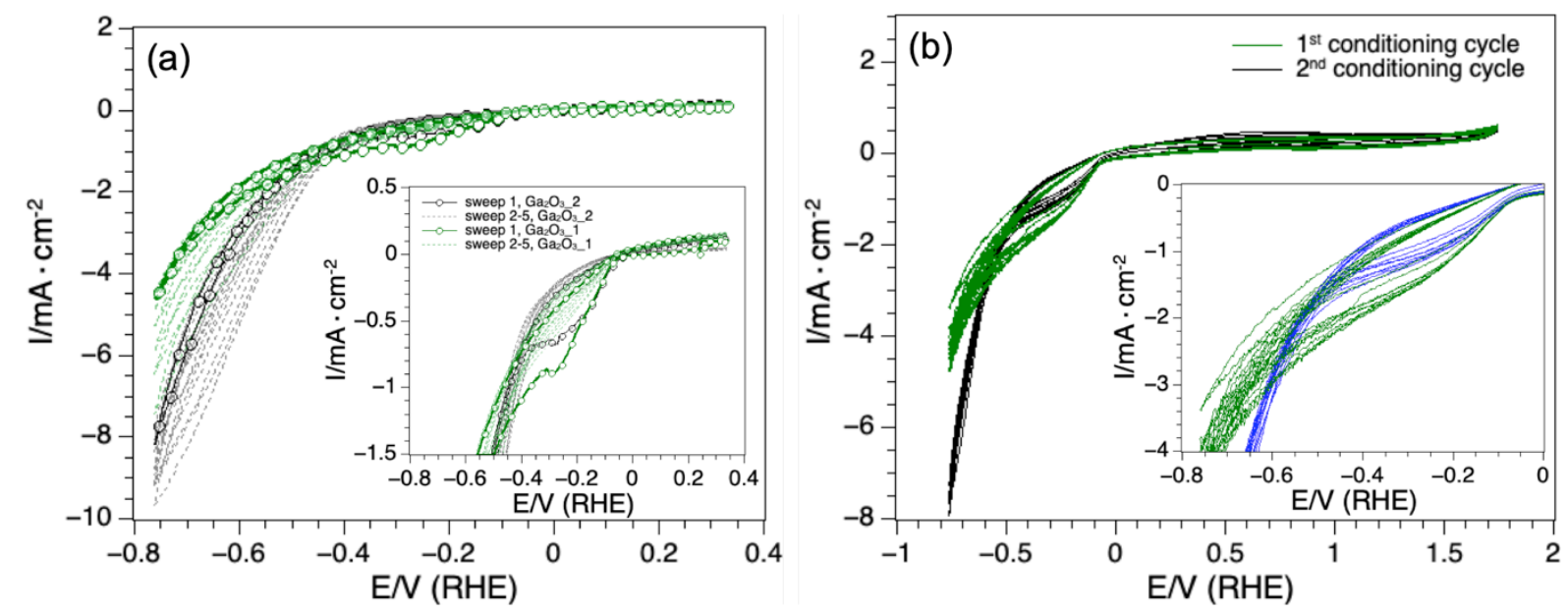

Fig. S8: Recorded cyclic voltammogram $(\mathrm{CV})$ traces of HER on $\mathrm{Ga}_{2} \mathrm{O}_{3}$ nanosheets after $1^{\text {st }}$ and $2^{\text {nd }}$ conditioning cycles; (b) Recorded CV traces of the conditioning cycles in the potential range -0.8 to $1.8 \mathrm{~V}$ vs. RHE. All CV traces were recorded under protective gas condition using nitrogen.

\section{Supplementary Figure 9}

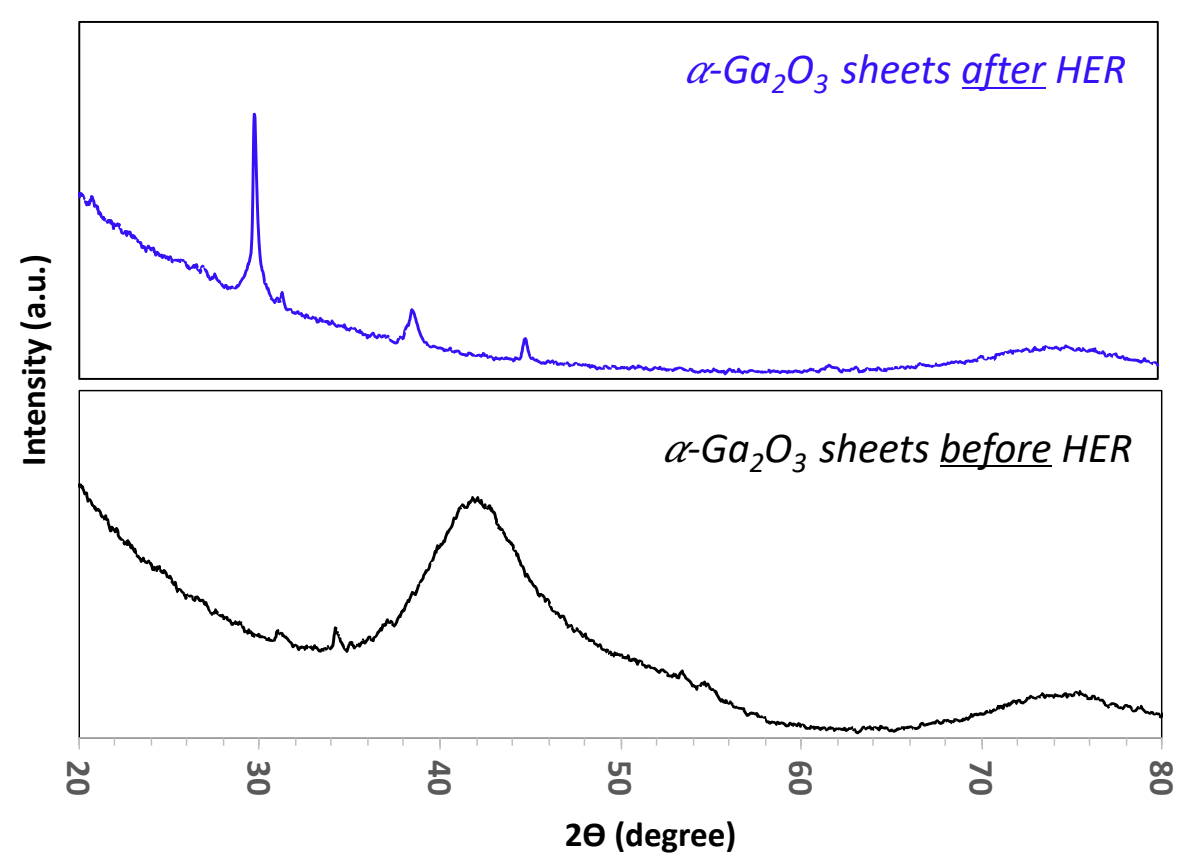

Fig. S9: XRD analysis before and after the electrocatalytic hydrogen evolution reaction (HER). The amorphous $\alpha-\mathrm{Ga}_{2} \mathrm{O}_{3}$ sheets synthesized in the VFD were observed to exhibit crystalline characteristics after electrocatalytic treatment. 


\section{Supplementary Table 1}

\begin{tabular}{|c|c|}
\hline Thickness (nm) & Average current at 6 V (nA) \\
\hline 5 & $>500$ \\
\hline 30 & 3 \\
\hline 100 & $0.05-0.1$ \\
\hline
\end{tabular}

Table S1: Average current at $6 \mathrm{~V}$ at different thicknesses of the $\mathrm{Ga}_{2} \mathrm{O}_{3}$ sheets

\section{References}

1. Elmas, S. et al. Copper Metallopolymer Catalyst for the Electrocatalytic Hydrogen Evolution Reaction (HER). Polymers 11. 
